\title{
Pion Transverse Momentum Spectrum, Elliptic Flow, and Interferometry in the Granular Source Model for RHIC and LHC Heavy Ion Collisions
}

\author{
Jing Yang, ${ }^{1}$ Yan-Yu Ren, ${ }^{2}$ and Wei-Ning Zhang ${ }^{1,2}$ \\ ${ }^{1}$ School of Physics and Optoelectronic Technology, Dalian University of Technology, Dalian, Liaoning 116024, China \\ ${ }^{2}$ Department of Physics, Harbin Institute of Technology, Harbin, Heilongjiang 150006, China \\ Correspondence should be addressed to Wei-Ning Zhang; wnzhang@dlut.edu.cn
}

Received 27 June 2014; Accepted 8 September 2014

Academic Editor: Bhartendu K. Singh

Copyright (C) 2015 Jing Yang et al. This is an open access article distributed under the Creative Commons Attribution License, which permits unrestricted use, distribution, and reproduction in any medium, provided the original work is properly cited. The publication of this article was funded by SCOAP ${ }^{3}$.

\begin{abstract}
We systematically investigate the pion transverse momentum spectrum, elliptic flow, and Hanbury-Brown-Twiss (HBT) interferometry in the granular source model for the heavy ion collisions of $\mathrm{Au}-\mathrm{Au}$ at $\sqrt{s_{N N}}=200 \mathrm{GeV}$ and $\mathrm{Pb}-\mathrm{Pb}$ at $\sqrt{s_{N N}}=$ $2.76 \mathrm{TeV}$ with different centralities. The granular source model can well reproduce the experimental results of the heavy ion collisions at the Relativistic Heavy Ion Collider (RHIC) and the Large Hadron Collider (LHC). We examine the parameters involved in the granular source model. The experimental data of the momentum spectrum, elliptic flow, and HBT radii for the two collision energies and different centralities impose very strict constraints on the model parameters. They exhibit certain regularities for collision centrality and energy. The space-time structure and expansion velocities of the granular sources for the heavy ion collisions at the RHIC and LHC energies with different centralities are investigated.
\end{abstract}

\section{Introduction}

The main purpose of relativistic heavy ion collisions is to probe the new matter, quark-gluon plasma (QGP), and study its properties. Because of the complexity of the nucleusnucleus collisions, model investigation plays important roles in determining and characterizing the QGP. Single particle spectrum, elliptic flow, and two-particle Hanbury-BrownTwiss (HBT) correlations are crucial final-state observables in relativistic heavy ion collisions [1-28]. They reflect the characteristics of the particle-emitting sources in different aspects at different stages. A combined investigation of these observables can provide very strong constraints for the source models. So far, much progress has been made in understanding the experimental data of the heavy ion collisions at the top energies of the Relativistic Heavy Ion Collider (RHIC) [29-57]. However, more detailed investigations of the physics beneath the data through multiobservable analyses are still needed. On the other hand, the experimental data of several $\mathrm{TeV}$ heavy ion collisions at the Large Hadron Collider
(LHC) have been recently published [15-25, 58-63]. It is an ambitious goal for the models to explain consistently the data of the heavy ion collisions at the RHIC and LHC energies.

In [64-67], Zhang et al. proposed and developed a granular source model of QGP droplets to explain the HBT data of the RHIC experiments [9-14]. Their investigations indicate that the short evolution lifetime and wide initial distribution of the QGP droplets in the granular source can lead to the result of the HBT radii, $R_{\text {out }} \sim R_{\text {side }}$. Here the labels "out" and "side" denote the transverse directions parallel and perpendicular to the transverse momentum of the pion pair [6870]. The results of the pion transverse momentum spectrum $[65,66]$, elliptic flow [65], and HBT radii $[65,66]$ of granular source are well in agreement with the experimental data of the pion momentum spectrum and elliptic flow for the $\mathrm{Au}$ Au collisions with minimum bias at $\sqrt{s_{N N}}=200 \mathrm{GeV}$ at the RHIC $[2,6]$ and the pion HBT measurements for the RHIC Au-Au collisions with $0-30 \%$ centrality $[11,12]$, respectively. They also find $[66,67]$ that the granular source model may reproduce the main characteristics of the two-pion source 
functions, extracted by the imaging techniques [71-75], in the RHIC experiments [13, 14]. In [76, 77], the fluctuating signatures of the single-event HBT correlation functions of granular sources are investigated. The detection of source inhomogeneity through the fluctuating single-event HBT correlation functions is discussed in [78], with the smoothed particle hydrodynamics (SPH) $[79,80]$. Recently, the HBT analyses in the granular source model for the experimental data of the most central $\mathrm{Pb}-\mathrm{Pb}$ collisions at $\sqrt{s_{N N}}=2.76 \mathrm{TeV}$ at the LHC [24] are performed [81]. The model parameters of the granular sources for the most central collisions at the RHIC and LHC energies are compared and discussed [81].

Although the granular source model reproduced the experimental data of the pion HBT radii for the Au-Au collisions in a certain centrality region at the RHIC and explained the pion $\mathrm{HBT}$ radii in the most central $\mathrm{Au}-\mathrm{Au}$ and $\mathrm{Pb}-\mathrm{Pb}$ collisions at the RHIC and the LHC $[65,66,81]$, it is still a challenge to models to explain consistently the experimental HBT measurements in different centrality regions of the collisions at the RHIC and the LHC energies. On the other hand, pion momentum spectrum and elliptic flow are very sensitive to collision centrality. A combined investigation of pion momentum spectrum, elliptic flow, and HBT interferometry in different centrality regions of the heavy ion collisions at the RHIC and the LHC energies, in the granular source model, is of great interest. In this work, we systematically investigate the pion transverse momentum spectrum, elliptic flow, and HBT interferometry in the granular source model for the heavy ion collisions at the RHIC and LHC energies with different centralities. In the combined investigations of pion momentum spectrum, elliptic flow, and HBT interferometry, we choose the experimental data in the centrality regions 0 $5 \%, 10-20 \%$, and $30-50 \%$ for the Au-Au collisions at $\sqrt{s_{N N}}=$ $200 \mathrm{GeV}$ at the RHIC $[1,2,7,12]$ and in the centrality regions $10-20 \%$ and $40-50 \%$ for the $\mathrm{Pb}-\mathrm{Pb}$ collisions at $\sqrt{s_{N N}}=$ $2.76 \mathrm{TeV}$ at the LHC $[17,22,25]$, because in these centrality regions the data of pion transverse momentum spectrum, elliptic flow, and HBT radii are available together. By comparing the granular source results of pion transverse momentum spectrum, elliptic flow, and HBT radii with the experimental data, we obtain the model parameters as a function of collision centrality and energy. We investigate the spacetime structure and expansion velocities of the granular sources at the RHIC and LHC energies with the different centralities. Our investigations indicate that the granular source model can reproduce the experimental data of the pion transverse momentum spectra, elliptic flow, and HBT radii of the $\mathrm{Au}-\mathrm{Au}$ collisions at the RHIC and the $\mathrm{Pb}-\mathrm{Pb}$ collisions at the LHC. The parameters in the granular source model exhibit certain regularities for collision centrality and energy. The space-time structure and expansion velocities of the granular source are consistent with that reflected by the observables.

The rest of this paper is arranged as follows. In Section 2, we describe the basic ingredients of the granular source model used in this work. In Section 3, we present the pion transverse momentum spectrum, elliptic flow, and HBT results of the granular sources for the heavy ion collisions at the RHIC and LHC energies with different centralities. The regularities of the model parameters are also discussed in this section. In Section 4, we investigate the space-time structure and expansion velocities of the granular sources. Finally, the summary and discussions are given in Section 5.

\section{Granular Source Model}

In the heavy ion collisions at the RHIC top energies and the LHC energy, the created strong-coupled QGP (sQGP) systems in the central rapidity region may reach local equilibrium at a very short time and then expand rapidly along the beam direction ( $z$-axis). Because of the random variations in the distribution of collision nucleons due to quantum fluctuations, the local equilibrium system is not uniform in the transverse plane ( $x-y$ plane) [82]. It may form many tubes along the beam direction during the subsequent fast longitudinal expansion and finally fragment into many QGP droplets with the effects of "sausage" instability, surface tension, and bulk viscosity [65, 83-85]. As a first-step idealized approximation, granular source model regards the whole source evolution as the superposition of the individual evolutions of the QGP droplets. Each droplet has a position-dependent initial velocity and evolves hydrodynamically.

As in [65], we suppose the QGP droplets in the granular source initially distribute within a cylinder along $z$-axis by

$$
\begin{aligned}
\frac{d N_{d}}{d x_{0} d y_{0} d z_{0}} \propto & {\left[1-e^{-\left(x_{0}^{2}+y_{0}^{2}\right) / \Delta \mathscr{R}_{T}^{2}}\right] \theta\left(\mathscr{R}_{T}-\rho_{0}\right) } \\
\times & \times\left(\mathscr{R}_{z}-\left|z_{0}\right|\right),
\end{aligned}
$$

where $\rho_{0}=\sqrt{x_{0}^{2}+y_{0}^{2}}$ and $z_{0}$ are the initial transverse and longitudinal coordinates of the droplet centers. The parameters $\mathscr{R}_{T}$ and $\mathscr{R}_{z}$ describe the initial sizes of the source, and $\Delta \mathscr{R}_{T}$ is a shell parameter in the droplet frame [65].

In [81], the Bjorken hypothesis [86] is used to describe the longitudinal velocity of droplet for the most central collisions, and the transverse velocity of droplet has a form of exponential power. Considering the longitudinal velocity of droplet varying with collision centrality, we also introduce a longitudinal power parameter, which will be determined by experimental data, to describe the longitudinal velocity phenomenologically. The initial velocities of the droplets in granular source frame are assumed as [65]

$$
v_{d i}=\operatorname{sign}\left(r_{0 i}\right) \cdot a_{i}\left(\frac{\left|r_{0 i}\right|}{\mathscr{R}_{i}}\right)^{b_{i}}, \quad i=1,2,3,
$$

where $r_{0 i}$ is $x_{0}, y_{0}$, or $z_{0}$ for $i=1,2$, or 3 , and $\operatorname{sign}\left(r_{0 i}\right)$ denotes the signal of $r_{0 i}$, which ensures an outward droplet velocity. In (2), $\mathscr{R}_{i}=\left(\mathscr{R}_{T}, \mathscr{R}_{T}, \mathscr{R}_{z}\right), a_{i}=\left(a_{x}, a_{y}, a_{z}\right)$, and $b_{i}=\left(b_{x}, b_{y}, b_{z}\right)$ are the magnitude and exponent parameters in $x, y$, and $z$ directions, which are associated with the early thermalization and pressure gradients of the system at the fragmentation. It is convenient to use the equivalent parameters $\bar{a}_{T}=$ $\left(a_{x}+a_{y}\right) / 2$ and $\Delta a_{T}=a_{x}-a_{y}$ instead of $a_{x}$ and $a_{y}$. The parameters $\bar{a}_{T}$ and $\Delta a_{T}$ describe the transverse expansion and asymmetric dynamical behavior of the system at the fragmentation, respectively. For simplicity, we take $b_{x}=b_{y}=b_{T}$ 
in calculations. The parameters $b_{T}$ and $b_{z}$ describe the coordinate dependence of exponential power in transverse and longitudinal directions.

In the calculations of the hydrodynamical evolution of the droplet, we use the equation of state (EOS) of the S95pPCE165-v0 [87], which combines the lattice QCD data at high temperature with the hadron resonance gas at low temperature. We assume systems fragment when reaching a certain local energy density and take the initial energy density of the droplets to be $2.2 \mathrm{GeV} / \mathrm{fm}^{3}$ for all considered collisions for simplicity [81]. The initial droplet radius is supposed satisfying a Gaussian distribution with the standard deviation $\sigma_{d}=2.5 \mathrm{fm}$ in the droplet local frame [81].

The final identical pions are considered to be emitted out of the surfaces of droplets with momenta obeying the BoseEinstein distribution in the local frame at freeze-out temperature $T_{f}$. To include the resonance decayed pions later as well as the directly produced pions at chemical freeze-out early, a wide region of $T_{f}$ is considered with the probability [81]

$$
\begin{aligned}
\frac{d P}{d T_{f}} \propto & f_{\text {dir }} e^{-\left(T_{\text {chem }}-T_{f}\right) / \Delta T_{\text {dir }}}+\left(1-f_{\text {dir }}\right) \\
& \times e^{-\left(T_{\text {chem }}-T_{f}\right) / \Delta T_{\text {dec }}}, \quad\left(T_{\text {chem }}>T_{f}>80 \mathrm{MeV}\right),
\end{aligned}
$$

where $f_{\text {dir }}$ is the fraction of the direct emission around the chemical freeze-out temperature $T_{\text {chem }} ; \Delta T_{\text {dir }}$ and $\Delta T_{\text {dec }}$ are the temperature widths for the direct and decay emissions, respectively. In the calculations, we take $f_{\text {dir }}=0.75, \Delta T_{\text {dir }}=$ $10 \mathrm{MeV}$, and $\Delta T_{\mathrm{dec}}=90 \mathrm{MeV}$ as in [81]. The value of $T_{\text {chem }}$ is taken to be $165 \mathrm{MeV}$ as it is taken in the S95p-PCE165-v0 EOS [87].

After fixing the parameters used in the calculations of hydrodynamical evolution and freeze-out temperature, the free model parameters are the three source geometry parameters $\left(R_{T}, \Delta R_{T}\right.$, and $\left.R_{z}\right)$ and the five droplet velocity parameters $\left(\bar{a}_{T}, \Delta a_{T}, a_{z}, b_{T}\right.$, and $\left.b_{z}\right)$. They are associated with the initial size, expansion, and directional asymmetry of system and have significant influence on the observables of pion momentum spectra, elliptic flow, and HBT radii. We will combine the experimental data of these observables to investigate the parameters of the granular source as a function of the collision centrality and energy in the heavy ion collisions at the RHIC and LHC energies in next sections.

\section{Pion Momentum Spectrum, Elliptic Flow, and HBT Results}

In high energy heavy ion collisions, the invariant momentum distribution of final particles can be written in the form of a Fourier series $[88,89]$

$$
E \frac{d^{3} N}{d^{3} p}=\frac{1}{2 \pi} \frac{d^{2} N}{p_{T} d p_{T} d y}\left[1+\sum_{n} 2 v_{n} \cos (n \phi)\right],
$$

where $E$ is the energy of the particle, $p_{T}$ is the transverse momentum, $y$ is the rapidity (it should not bring a mistake with coordinate from the context), and $\phi$ is the azimuthal angle with respect to the reaction plane. In (4), the first term on the right is the transverse momentum spectrum in the rapidity region $d y$, and the second harmonic coefficient $v_{2}$ in the summation is called elliptic flow.

At the RHIC and LHC energies, the spectators in nucleusnucleus collisions depart from the reaction region rapidly after collision, and a very hot and dense fireball is formed in an almond shape perpendicular to the reaction plane. The particle spectra of transverse momentum at low $p_{T}\left(p_{T} \lesssim\right.$ $3 \mathrm{GeV} / c$ ) contain the information about the transverse expansion and thermal properties of the particle-emitting sources at freeze-out temperature [15-19]. By comparing the pion transverse momentum spectra of the granular sources with experimental data, we can constrain the velocity parameters $\bar{a}_{T}$ and $a_{z}$ of the granular sources.

Choosing $x$ axis on the reaction plane, elliptic flow $v_{2}$ can be expressed as

$$
v_{2}\left(p_{T}\right)=\langle\cos (2 \phi)\rangle=\left\langle\frac{p_{x}^{2}-p_{y}^{2}}{p_{T}^{2}}\right\rangle .
$$

Since the reaction plane orientation is hard to estimate exactly in experiment, an alternative technique for elliptic flow analysis is the measurement of the two-particle cumulant of azimuthal correlations, $v_{2}^{2}\{2\}[4,5,7,8,20-23,90]$, which gives essentially the same results as the reaction-plane method $[4,7,8,91]$.

In noncentral nucleus-nucleus collisions, the initial space-asymmetry of the system can bring anisotropic pressure gradients, which lead to the anisotropic transversemomentum distributions of final particles and nonzero $v_{2}$. For the granular source, the results of elliptic flow are very sensitive to the parameters $\Delta a_{T}, b_{T}$, and $b_{z}$ [65]. The experimental data of the transverse momentum spectrum and elliptic flow impose strict constraints on the velocity parameters in the granular source model.

Two-particle Hanbury-Brown-Twiss (HBT) correlation function is defined as the ratio of the two-particle momentum spectrum $P\left(\mathbf{p}_{1}, \mathbf{p}_{2}\right)$ to the product of two single-particle momentum spectra $P\left(\mathbf{p}_{1}\right) P\left(\mathbf{p}_{2}\right)$. It has been widely used to extract the space-time geometry and dynamic and coherence information of the particle-emitting source in high energy heavy ion collisions [92-96]. In the usual HBT analysis in high energy heavy ion collisions, the two-pion correlation functions are fitted by the Gaussian parameterized formula

$$
C\left(q_{\text {out }}, q_{\text {side }}, q_{\text {long }}\right)=1+\lambda e^{-R_{\text {out }}^{2} q_{\text {out }}^{2}-R_{\text {side }}^{2} q_{\text {side }}^{2}-R_{\text {long }}^{2} q_{\text {long }}^{2}}
$$

where $q_{\text {out }}, q_{\text {side }}$, and $q_{\text {long }}$ are the Bertsch-Pratt variables, which denote the components of the relative momentum, $\mathbf{q}=\mathbf{p}_{1}-\mathbf{p}_{2}$, in the "out" (parallel to the momentum of the particle pair in transverse plane), "side" (perpendicular to the momentum of the particle pair in transverse plane), and longitudinal directions [68-70], respectively. In (6) $\lambda$ is chaoticity parameter of source, $R_{\text {out }}, R_{\text {side }}$, and $R_{\text {long }}$ are the HBT radii in out, side, and long directions. The results of HBT radii are related to the source geometry as well as expansion. We can finally determine the geometry parameters $\left(R_{T}, \Delta R_{T}\right.$, and $R_{z}$ ) and the velocity parameters $\left(\bar{a}_{T}, \Delta a_{T}, a_{z}, b_{T}\right.$, and $\left.b_{z}\right)$ in the 


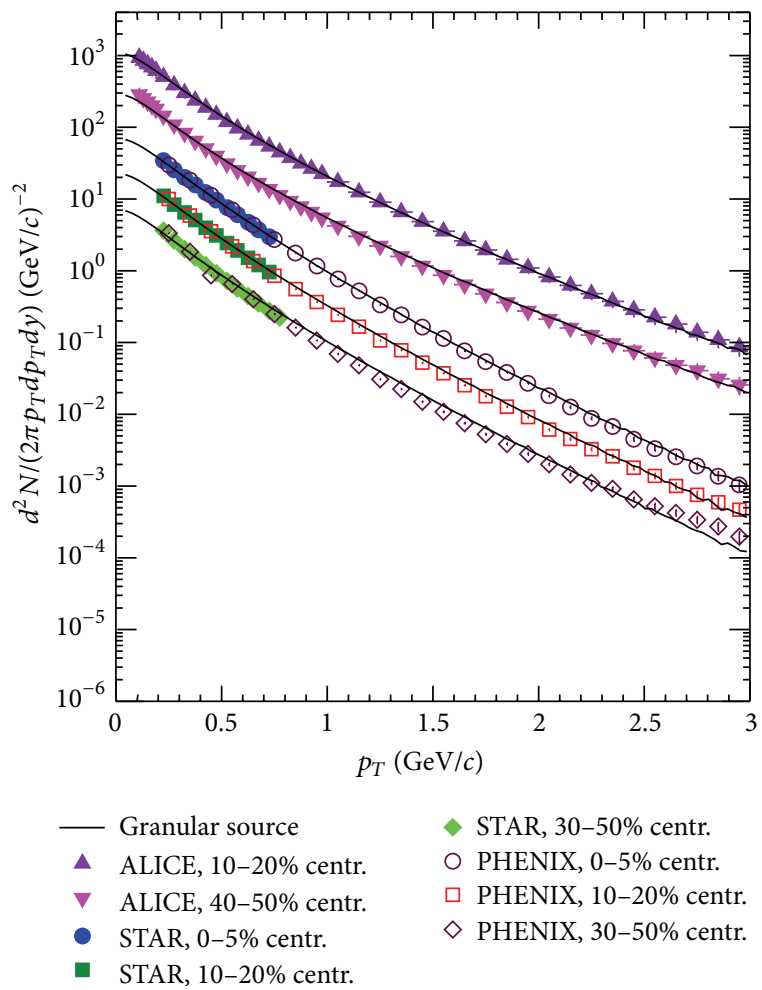

(a)

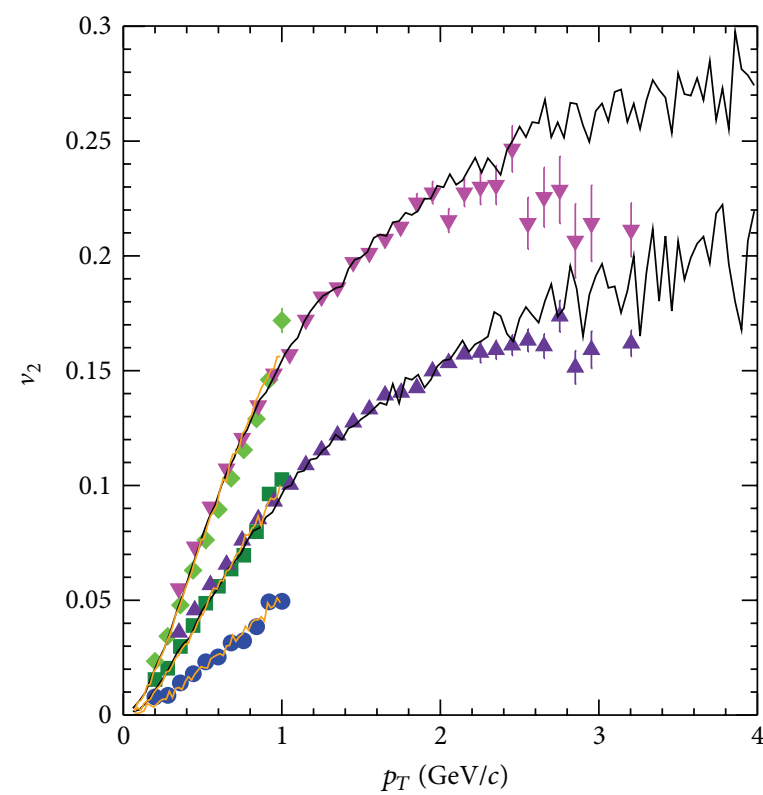

$\begin{array}{lll}\text { - Granular source (STAR) } & \diamond & \text { STAR, } 30-50 \% \text { centr. } \\ \text { - Granular source (ALICE) } & \text { ALICE, } 10-20 \% \text { centr. } \\ \text { - STAR, } 0-5 \% \text { centr. } & \nabla \text { ALICE, } 40-50 \% \text { centr. } \\ \text { - STAR, } 10-20 \% \text { centr. } & \end{array}$

(b)

Figure 1: (Color online) (a) the pion transverse momentum spectra of the granular sources (solid lines) and the experimental data of negative pions in the Au-Au collisions at $\sqrt{s_{N N}}=200 \mathrm{GeV}[1,2]$ and the Pb-Pb collisions at $\sqrt{s_{N N}}=2.76 \mathrm{TeV}$ [17]. (b) The elliptic flow of the granular source (solid lines) and the experimental data of negative pions in the Au-Au collisions at $\sqrt{s_{N N}}=200 \mathrm{GeV}$ [7] and the Pb-Pb collisions at $\sqrt{s_{N N}}=2.76 \mathrm{TeV}$ [22].

granular source model by the multiobservable analyses of the pion spectrum, elliptic flow, and HBT interferometry.

In the multiobservable analyses, we choose the experimental data of the Au-Au collisions at $\sqrt{s_{N N}}=200 \mathrm{GeV}$ at the RHIC $[1,2,7,12]$ and the Pb-Pb collisions at $\sqrt{s_{N N}}=2.76 \mathrm{TeV}$ at the LHC $[17,22,25]$ for determining the geometry and velocity parameters of the granular sources. These experimental data provide the identical pion $p_{T}$ spectrum, elliptic flow, and HBT radii simultaneously in the same centrality regions (for RHIC: $0-5 \%, 10-20 \%$, and 30-50\%; for LHC: $10-$ $20 \%$ and $40-50 \%)$.

In Figure 1, we plot the pion transverse momentum spectra (a) and elliptic flow (b) of the granular sources for the heavy ion collisions at the RHIC and LHC energies with different centralities. The experimental data of the pion transverse momentum spectra and elliptic flow of the $\mathrm{Au}-$ Au collisions with $0-5 \%, 10-20 \%$, and $30-50 \%$ centralities at $\sqrt{s_{N N}}=200 \mathrm{GeV}$ at the RHIC $[1,2,7]$ and the $\mathrm{Pb}-\mathrm{Pb}$ collisions with $10-20 \%$ and $40-50 \%$ centralities at $\sqrt{s_{N N}}=$ $2.76 \mathrm{TeV}$ at the LHC $[17,22]$ are shown simultaneously. Complying with the experimental measurements, we use the rapidity cuts $|y|<0.1[1]$ and $|y|<0.5[16,17]$ in the calculations of the $p_{T}$ spectra of the granular sources at the RHIC energy and the LHC energy, respectively. In the calculations of the elliptic flow of the granular sources, the pseudorapidity cuts $|\eta|<1.0$ and $|\eta|<0.8$ are adopted as the same as in the experimental analyses at the RHIC [7] and LHC [22], respectively.

As shown in Figure 1, the pion transverse momentum spectra of the granular sources agree with the experimental data with different centralities at the RHIC and LHC energies simultaneously. The spectra at the LHC energy exhibit clear upwarp at $p_{T}>1.5 \mathrm{GeV} / c$ as compared to those at the RHIC energy, because the average transverse velocities of the LHC sources are larger. The results of elliptic flow at the LHC energy almost match the elliptic flow results at the RHIC energy with the same and near centrality regions. The results of elliptic flow of granular sources exhibit clear centrality dependence as the same that the experimental data has [7, $22]$. The $v_{2}$ results decrease with increasing collision centrality. At $p_{T}>2.5 \mathrm{GeV} / c$, the granular source results of elliptic flow are a little higher than those of experimental data. It reflects the limitations of the model based on hydrodynamical evolution to describe the particles with high $p_{T}$, which originate from the early stage with incomplete thermalization and have a relative small anisotropic characteristic in the transverse momentum space [97-99].

In Figure 2, we show the granular source results of the pion HBT radii and chaoticity parameter as a function of the transverse momentum of pion pair, $k_{T}=\left|\mathbf{p}_{1}+\mathbf{p}_{2}\right| / 2$, obtained by Gaussian parameterized formula fit in the longitudinally comoving system (LCMS) $[94,96]$. The experimental data of STAR Au-Au collisions at $\sqrt{s_{N N}}=200 \mathrm{GeV}$ 


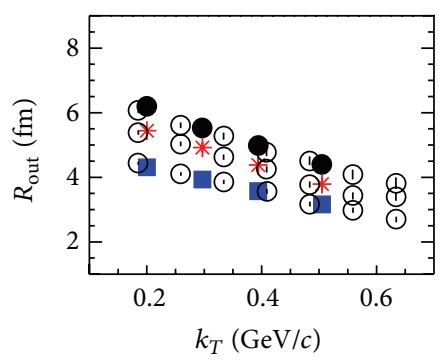

(a)

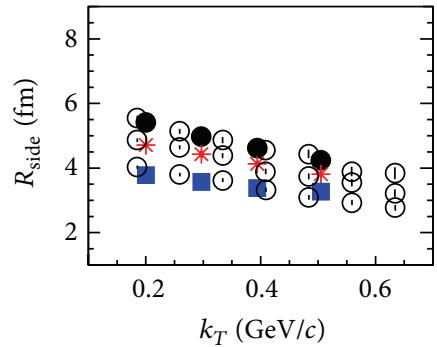

(b)

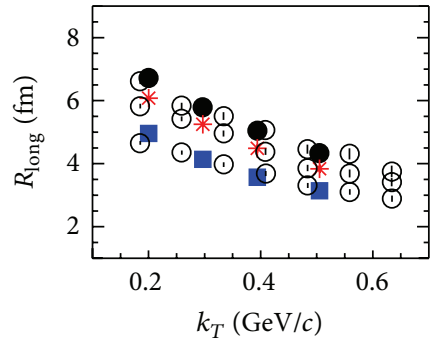

(c)

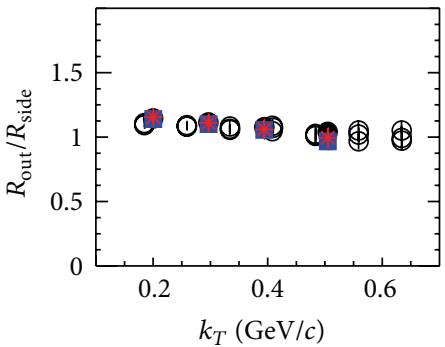

(d)

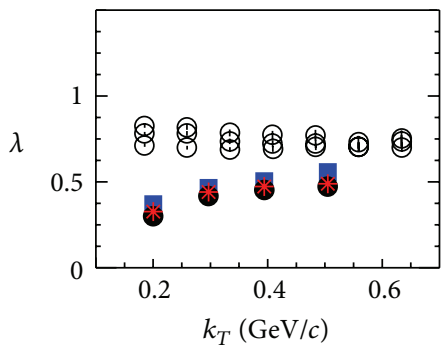

(e)

- STAR, $0-5 \%$ centr.

* STAR, $10-20 \%$ centr.

- STAR, 30-50\% centr.

$\bigcirc$ Granular source

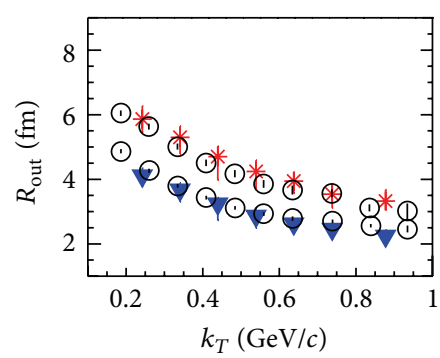

$\left(\mathrm{a}^{\prime}\right)$

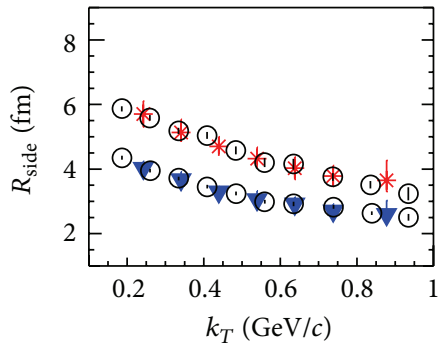

$\left(b^{\prime}\right)$

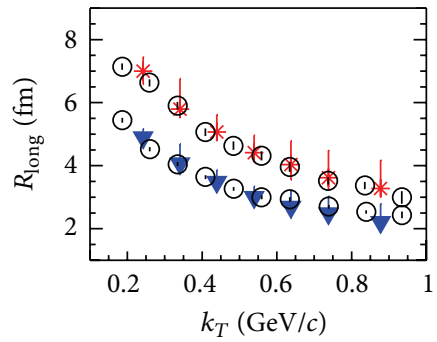

(c')

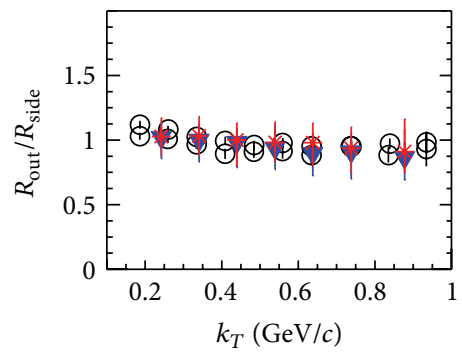

$\left(d^{\prime}\right)$

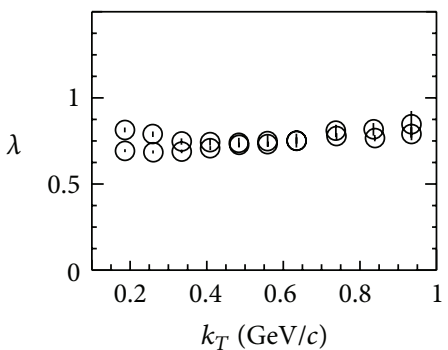

$\left(\mathrm{e}^{\prime}\right)$

* ALICE, 10-20\% centr.

ALICE, $40-50 \%$ centr.

O Granular source

FIGURE 2: (Color online) the results of pion HBT radii and chaoticity parameter of the granular sources (open circle) with different centralities for the highest RHIC energy (left column) and LHC energy (right column). The solid circle, star, and solid diamond symbols in the left column are the STAR data of the Au-Au collisions at $\sqrt{s_{N N}}=200 \mathrm{GeV}$ with $0-5 \%, 10-20 \%$, and $30-50 \%$ centralities [12], respectively. The star and solid triangle-down symbols in the right column are the ALICE data of the Pb-Pb collisions at $\sqrt{s_{N N}}=2.76 \mathrm{TeV}$ with $10-20 \%$ and $40-50 \%$ centralities [25], respectively. 
TABLE 1: The geometry and velocity parameters of the granular sources.

\begin{tabular}{lcccccccc}
\hline Centrality & $\mathscr{R}_{T}(\mathrm{fm})$ & $\Delta \mathscr{R}_{T}(\mathrm{fm})$ & $\mathscr{R}_{z}(\mathrm{fm})$ & $\bar{a}_{T}$ & $\Delta a_{T}$ & $a_{z}$ & $b_{T}$ & $b_{z}$ \\
\hline RHIC, 0-5\% & 5.8 & 0.7 & 3.9 & 0.469 & 0.066 & 0.593 & 0.76 & 0.13 \\
RHIC, 10-20\% & 4.5 & 0.5 & 2.9 & 0.454 & 0.115 & 0.593 & 0.56 & 0.11 \\
RHIC, 30-50\% & 2.5 & 0.3 & 0.5 & 0.437 & 0.156 & 0.593 & 0.37 & 0.06 \\
\hline LHC, 10-20\% & 6.0 & 0.9 & 5.5 & 0.431 & 0.092 & 0.592 & 0.35 \\
LHC, 40-50\% & 2.5 & 0.4 & 1.8 & 0.407 & 0.131 & 0.590 & 0.23 & 0.03 \\
\hline
\end{tabular}

[12] and ALICE Pb-Pb collisions at $\sqrt{s_{N N}}=2.76 \mathrm{TeV}$ [25], which have the same centralities as the experimental data of pion $p_{T}$ spectra and $v_{2}$ shown in Figure 1, are also plotted for comparing. In the HBT analyses of the granular sources for the RHIC and LHC energies, we applied the rapidity cut $|y|<0.5$ and the pseudorapidity cut $|\eta|<0.8$ as the same in the experimental analyses of STAR [12] and ALICE [25], respectively.

From Figure 2 it can be seen that the HBT radii $R_{\text {out}}$, $R_{\text {side }}$, and $R_{\text {long }}$ of the granular sources simultaneously agree with the experimental data at the RHIC and LHC energies. Both the transverse and longitudinal HBT radii increase with increasing collision centrality. At the LHC energy the results of the HBT radii $R_{\text {out }}, R_{\text {side }}$, and $R_{\text {long }}$ are larger than those at the RHIC energy, respectively. However, the results of the ratio of $R_{\text {out }} / R_{\text {side }}$ are always about 1 and independent of the collision centrality and energy. In Figure 2(e), we plot the results of the chaoticity parameter $\lambda$ of the granular sources and the RHIC experiments [12]. Because of absence of the experimental data of $\lambda$ in the $\mathrm{Pb}-\mathrm{Pb}$ collisions at the $\mathrm{LHC}$ [25], we plot in Figure 2( $\left.\mathrm{e}^{\prime}\right)$ only the results of the granular source. In experimental HBT measurements, many effects, such as the Coulomb interaction between the final particles, particle missing-identification, source coherence, and so forth, can influence the results of the chaoticity parameter $\lambda$ [92-96]. Because these effects are not considered in the granular source model, the $\lambda$ results of the granular sources are larger than the experimental data. The Coulomb interaction is greater for the particles with smaller relative momentum. It mainly influences the $\lambda$ value which is related to the HBT correlation functions at zero relative momentum, and its effect on the HBT radii, which are related to the widths of the HBT correlation functions, is smaller. In experimental HBT analyses, the Coulomb interaction between final identical particles must be considered [92-96].

In Table 1 we present the values of the geometry and velocity parameters of the granular sources used in the multiobservable analyses. One can see that the value of $\mathscr{R}_{T}$ for a certain collision centrality and energy is larger than that of $\mathscr{R}_{z}$. So the initial geometry of the granular source is a short cylinder. However, the ratio of $\mathscr{R}_{z}$ to $\mathscr{R}_{T}$ for the LHC sources becomes large for the noncentral collisions. It means the initial shape of the source changes from a short cylinder to a big cylinder with increasing collision energy. The initial transverse and longitudinal sizes of the granular sources $\mathscr{R}_{T}$ and $\mathscr{R}_{z}$ increase with increasing collision centrality. The transverse shell parameter $\Delta \mathscr{R}_{T}$ also increases with increasing collision centrality. Because $\Delta \mathscr{R}_{T} \ll \mathscr{R}_{T}$, the shell effect is small and the initial distributions of droplets are almost volume distribution. For $10-20 \%$ centrality, the values of the geometry parameters $\mathscr{R}_{T}$ and $\mathscr{R}_{z}$ of the granular source for the RHIC energy are smaller than that for the LHC energy, respectively. The QGP droplets in the granular sources initially distribute in larger transverse and longitudinal regions for the more central and higher energy collisions.

In transverse direction, the velocity parameters $\bar{a}_{T}$ and $b_{T}$ increase with increasing collision centrality. For a fixed $b_{T}$, the larger the parameter $\bar{a}_{T}$, the larger the average transverse velocity of droplet. Because the values of $b_{T}$ are less than one, the larger the $b_{T}$, the smaller the average transverse velocity of droplet, if $\bar{a}_{T}$ is fixed. In longitudinal direction, the parameter $a_{z}$ is almost independent of collision centrality and energy. The values of the parameter $b_{z}$ are much smaller than those of $b_{T}$, while $b_{z}$ increases with increasing collision centrality as $b_{T}$. The large difference between the values of the transverse and longitudinal exponent parameters $b_{T}$ and $b_{z}$ and the different centrality dependence of $\bar{a}_{T}$ and $a_{z}$ reflect the different dynamical behaviors in transverse and longitudinal directions in the heavy ion collisions at the RHIC and LHC energies. In Figure 3 we plot the droplet velocities $v_{d \rho}=$ $\bar{a}_{T}\left(\rho / \mathscr{R}_{T}\right)^{b_{T}}$ and $v_{d z}=a_{z}\left(|z| / \mathscr{R}_{z}\right)^{b_{z}}$ of the granular sources. One can see that the droplet transverse velocity of central collision is smaller than that of peripheral collision in the center region of the source, although the droplet transverse velocity of central collision is larger at the edge of the source. The average longitudinal velocity of droplet is larger than the average transverse velocity. At the LHC energy, the droplet longitudinal velocity for peripheral collision is almost a constant. And, it differs significantly for the different centralities. From Table 1, it can be seen that the velocity parameter $\Delta a_{T}$ decreases with collision centrality. This leads to the increase of $v_{2}$ with decreasing collision centrality.

\section{Granular Source Space-Time and Expansion}

In the granular source model, the results of single particle $p_{T}$ spectrum, elliptic flow, and HBT radii are strongly related to the source space-time and expansion properties. The successes of the granular source model in explaining the experimental data of pion $p_{T}$ spectrum, elliptic flow, and HBT radii in the heavy ion collisions at the RHIC and LHC inspire us to further study the granular source space-time and expansion features.

In Figure 4, we show the pictures of the distributions of pion source points in one granular source event with $4 \times 10^{5}$ 


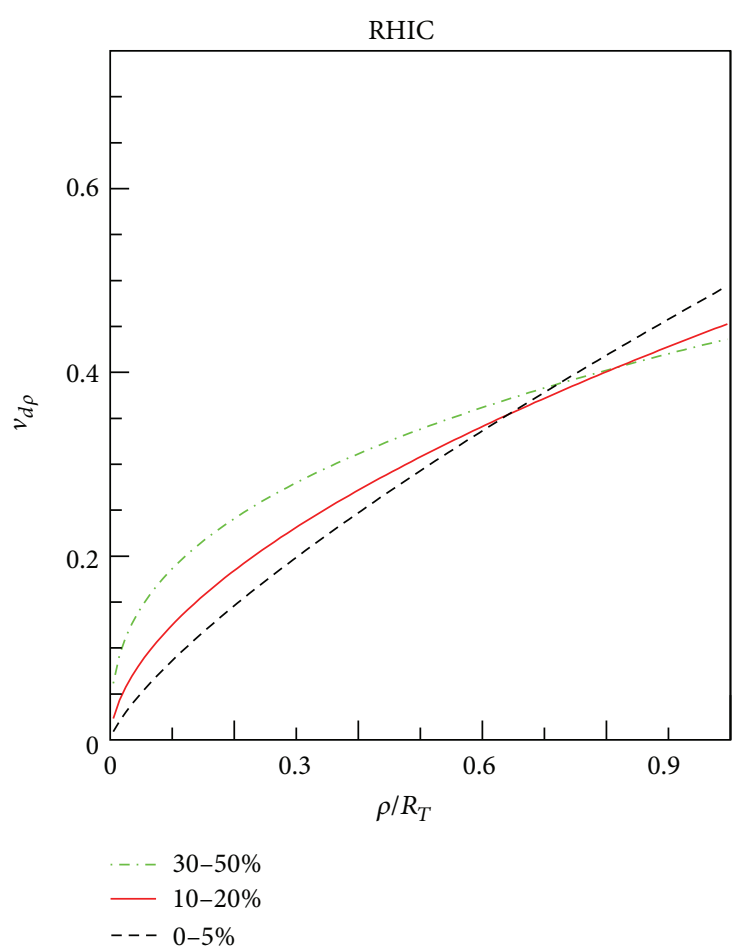

(a)

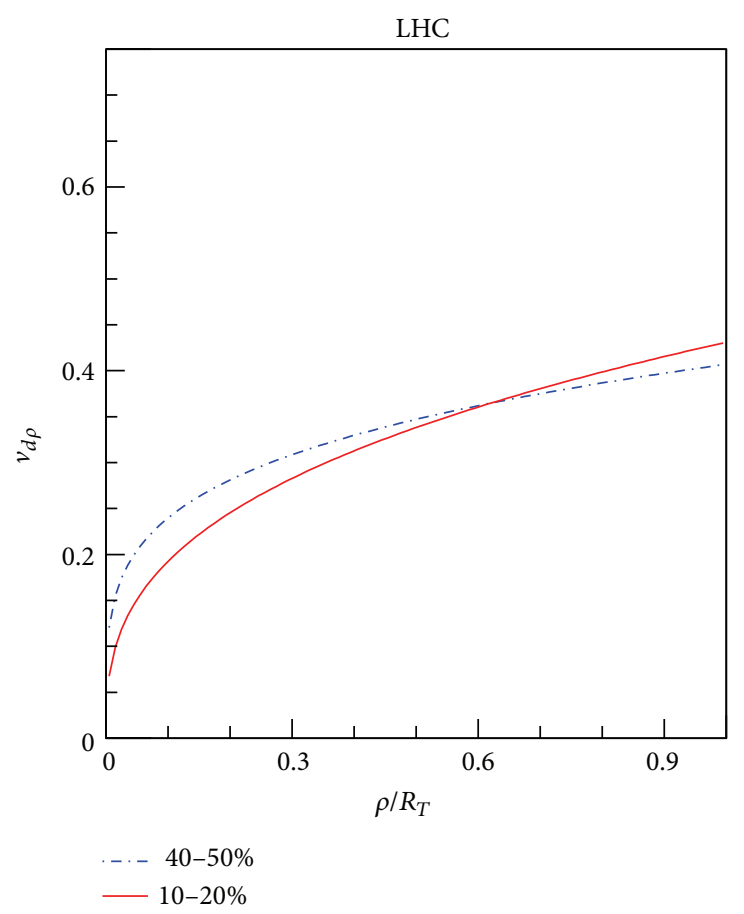

(c)

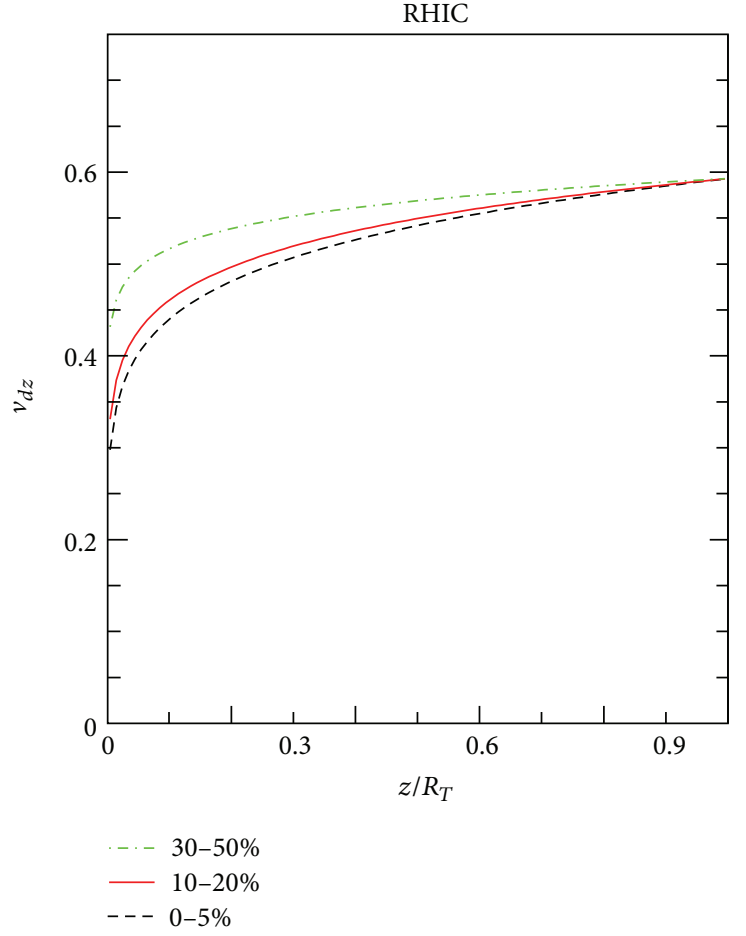

(b)

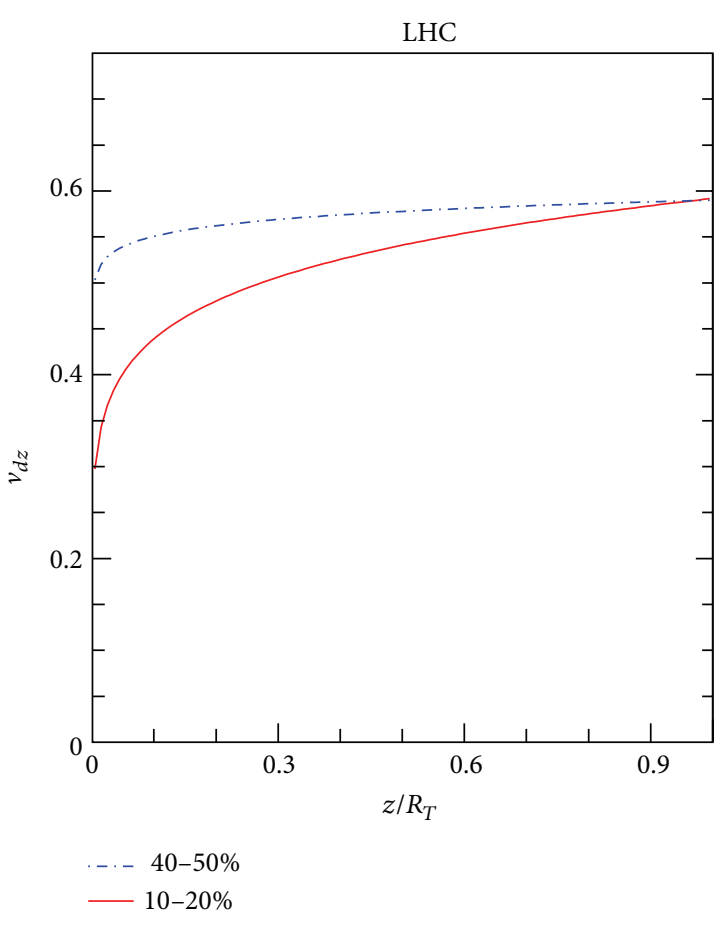

(d)

FIGURE 3: (Color online) the droplet transverse and longitudinal velocities $v_{d \rho}=\bar{a}_{T}\left(\rho / \mathscr{R}_{T}\right)^{b_{T}}$ and $v_{d z}=a_{z}\left(|z| / \mathscr{R}_{z}\right)^{b_{z}}$.

pion pairs for the heavy ion collisions at the RHIC energy with $0-5 \%$ centrality. The $z$ region for the $x-y$ distributions (panels (a), (b), and (c)) is $|z|<1 \mathrm{fm}$. The $y$ region for the $x-z$ distributions (panels (d), (e), and (f)) is $|y|<1 \mathrm{fm}$. The time for the panels (a) and (d), (b) and (e), and (c) and (f) is $t=4,8$, and $12 \mathrm{fm} / c$, respectively. For each picture the exposure time is $0.5 \mathrm{fm} / \mathrm{c}$. One can see that the distributions are inhomogeneous. There are separated "clumps," which correspond to the separated droplets, in the distributions. The clump-structure exists in the whole duration of source 


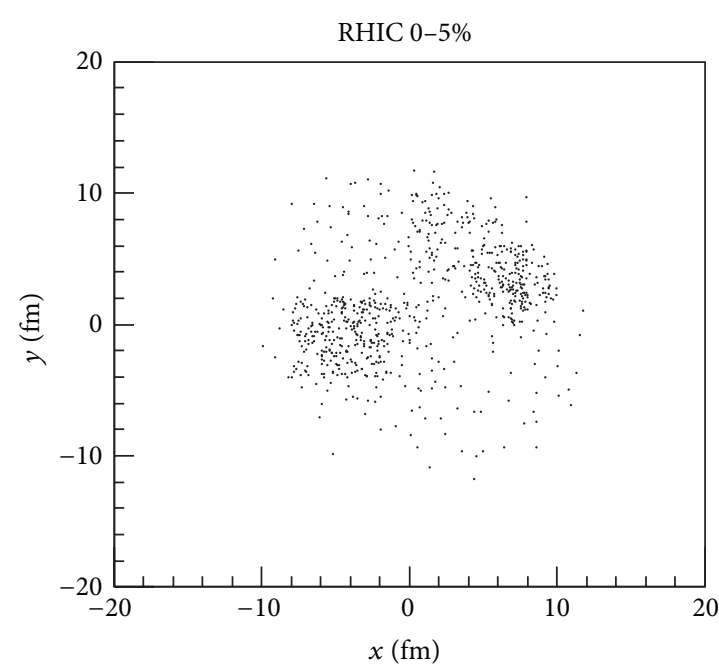

(a)

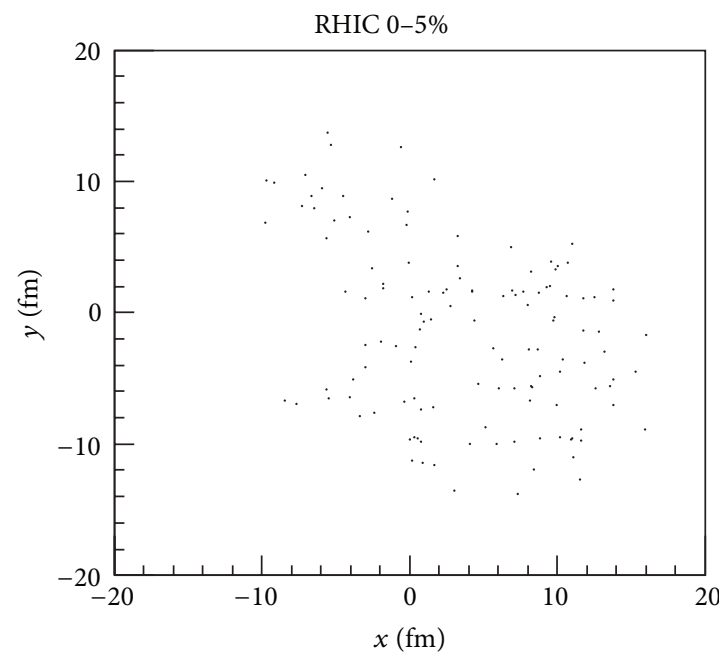

(c)

RHIC $0-5 \%$

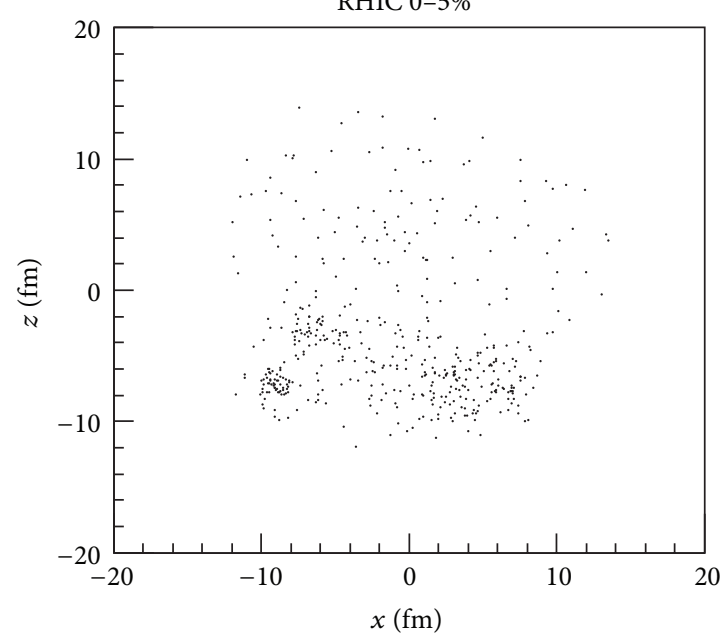

(e)

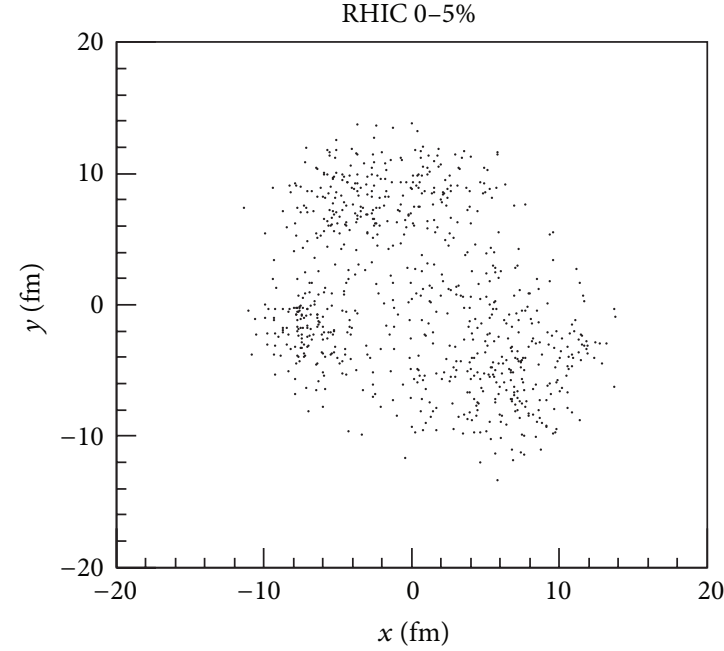

(b)

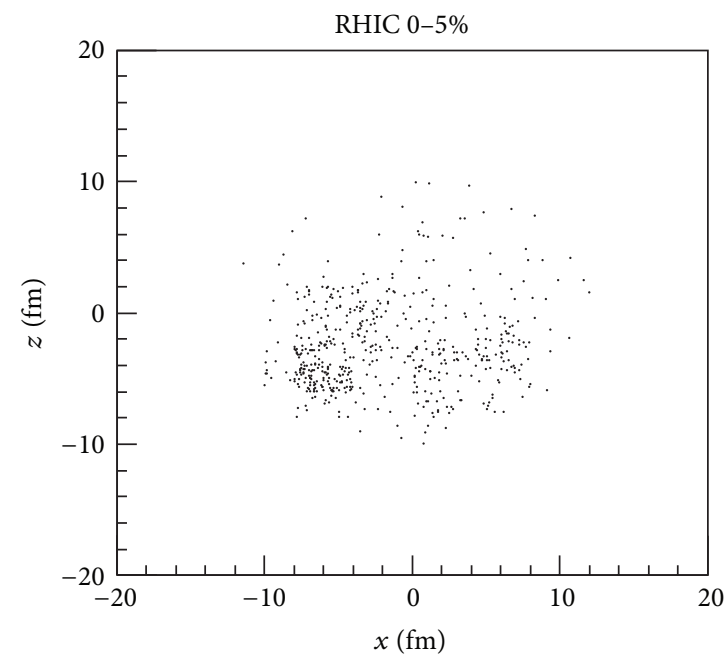

(d)

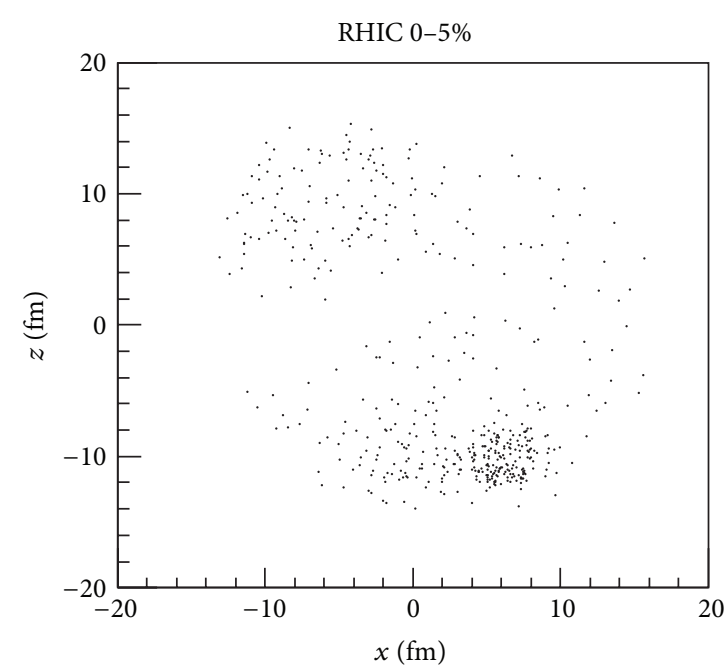

(f)

FIGURE 4: The pictures of the distributions of pion source points in one granular source event for the RHIC central collision. The $z$ region for the panels (a), (b), and (c) is $|z|<1 \mathrm{fm}$. The $y$ region for the panels (d), (e), and (f) is $|y|<1 \mathrm{fm}$. The time for the panels (a) and (d), (b) and (e), and (c) and (f) is $t=4,8$, and $12 \mathrm{fm} / c$, respectively. The exposure time for these pictures is $0.5 \mathrm{fm} / c$. 
evolution. Because the correlated two pions are taken from the same event in two-pion HBT analysis, the effect of the clump-structure on the HBT results will exist even for manyevent analysis, although the clump-structure may smooth out instead of a continued distribution of the source point for many events. Because many droplets evolve simultaneously in the granular source, the source lifetime is smaller as compared to that of a continued big source, which evolves in whole and freezes out from the source surface $[64,65,81,100$, 101]. It will be seen that the distributions of source points for many events are more volume distributions rather than surface distributions because of the contributions from the droplets in the central region of the granular source.

In Figure 5, we plot the space-time distributions of the pion source points of the granular sources projected on $t$ $r_{\text {out }}, t-r_{\text {side }}$, and $t-r_{\text {long }}$ planes, for five hundred events for the heavy ion collisions at the RHIC energy with $0-5 \%$ and $30-50 \%$ centralities. Here, the dashed lines are the average values of $r_{i}$ ( $i=$ out, side, long) and $t$ for all of the source points, and the bullets are the average values of $r_{i}$ obtained by averaging over the same $t$ bins. In the calculations the same rapidity cut $|y|<0.5$ as in the experimental HBT analyses [12] is used. We take the relative momentum cut $\left|q_{i}\right|<100 \mathrm{MeV} / c$ for pion pairs because most of the contributions in HBT correlation functions come from the particle pairs with small relative momenta [92-96]. One can see that the distributions for many events are smoothed. The widths of the $r_{i}$-distributions for the central collision are wider than those for the peripheral collision. In side and long directions, the distributions are symmetric with respect to $r_{i}=0$. However, one can observe a time-increased asymmetry for the distributions in out direction. It is because the coordinate-dependent source transverse expansion boosts particle momenta along the direction out of the source, and this coordinate-momentum correlation leads the result that the average angle between particle momentum and emitting coordinate trends to be smaller than isotropic emission. For the central and peripheral collisions, the average values of $r_{\text {out }}$ are 2.52 and $2.78 \mathrm{fm}$, respectively. The coordinate-momentum correlation for the peripheral is larger than that for the central collision. This is because the peripheral collision has a larger source transverse velocity as compared to the central collision (which will be seen in Figure 7(a)).

In Figure 6 we compare the space-time distributions of the pion source points of the granular sources for the collisions at the RHIC and LHC energies with the same centrality, $10-20 \%$. As in Figure 5 the dashed lines are for the average values of $r_{i}$ and $t$ for all of the source points, and the bullets are the average values of $r_{i}$ obtained from the same $t$ bins. We take the relative momentum cut $\left|q_{i}\right|<100 \mathrm{MeV} / c$ in the calculations. The rapidity and pseudorapidity cuts $|y|<$ 0.5 and $|\eta|<0.8$ are taken for the RHIC and LHC collisions, respectively, as in the experimental $\mathrm{HBT}$ analyses $[12,25]$. It can be seen that the widths of the $r_{\text {out }}, r_{\text {side }}$, and $r_{\text {long }}$ distributions for the LHC source are wider as compared to those for the RHIC source with the same collision centrality. In side and long directions, the distributions are symmetric with respect to $r_{i}=0$. But the distributions in out direction are asymmetric with respect to $r_{\text {out }}=0$ because of the coordinate-momentum correlations arising from the source transverse expansion. The asymmetry effect is larger for the granular source at the LHC energy $\left(\left\langle r_{\text {out }}\right\rangle=3.59 \mathrm{fm}\right)$ than that at the RHIC energy $\left(\left\langle r_{\text {out }}\right\rangle=2.64 \mathrm{fm}\right)$. This is because the granular source for the LHC collision has a larger transverse velocity as compared to that for the RHIC collision.

Figures 7(a) and 7(b) display the average transverse and longitudinal velocities of the granular sources as a function of time at the RHIC energy. Figures 7(c) and 7(d) display the average transverse and longitudinal velocities of the granular sources as a function of time at the LHC energy. It can be seen that the average velocities increase with increasing collision centrality at both the energies. The larger average transverse velocities of sources at the LHC energy are consistent with the results that the $p_{T}$ spectra at the LHC energy exhibit upwarp at larger $p_{T}$ as compared to those at the RHIC energy (see Figure 1(a)). The differences of the transverse velocities for different centralities become small at the higher energy, and the differences of the longitudinal velocities for different centralities are larger at the LHC energy. In Figures $7(\mathrm{e})$ and $7(\mathrm{f})$ we present the average transverse and longitudinal droplet velocities for the granular sources at the RHIC and LHC energies. The average transverse and longitudinal droplet velocities increase with the decreasing collision centrality for both the energies. In our granular source model, the droplet evolution in the local frame is independent of the collision energy and centrality. However, the different droplet velocities lead to the difference between the average emission time in the source center-of-mass frame because of the different Lorentz time delays. The larger the droplet velocity the larger the emission time. For example, the average emission time of the granular sources for the RHIC central and peripheral collisions is 8.39 and $9.11 \mathrm{fm} / c$, respectively (see Figure 5). The average emission time for the granular source for LHC peripheral collision is the largest because the average droplet velocity is the largest in this case (see Figure 7(e)).

In the two-pion interferometry in high energy heavy ion collisions, the difference between the transverse HBT radii $R_{\text {out }}$ and $R_{\text {side }}$ satisfies [94, 102, 103]

$$
R_{\text {out }}^{2}\left(k_{T}\right)-R_{\text {side }}^{2}\left(k_{T}\right) \approx\left[\left\langle\beta_{T} \tilde{t}^{2}\right\rangle-2\left\langle\beta_{T} \tilde{r}_{\text {out }} \tilde{t}\right\rangle\right]\left(k_{T}\right),
$$

where $\beta_{T}=\left|\mathbf{p}_{1 T}+\mathbf{p}_{2 T}\right| /\left(E_{1}+E_{2}\right)$ is the transverse velocity of the pair; $\tilde{t}=t-\langle t\rangle$ and $\widetilde{r}_{\text {out }}=r_{\text {out }}-\left\langle r_{\text {out }}\right\rangle$ are the deviations of source time and coordinate $r_{\text {out }}$ from their averages, respectively.

In Figure 8, we plot $\left\langle\beta_{T}^{2} \widetilde{t}^{2}\right\rangle, 2\left\langle\beta_{T} \widetilde{r}_{\text {out }} \tilde{t}\right\rangle$, and the average transverse and longitudinal velocities of the pion pair as a function of $k_{T}$. It can be seen that $\left\langle\beta_{T}^{2} \widetilde{t}^{2}\right\rangle$ and $2\left\langle\beta_{T} \widetilde{r}_{\text {out }} \widetilde{t}\right\rangle$ increase as $k_{T}$ increases. At small $k_{T}$, the values of $\left\langle\beta_{T}^{2} \widetilde{t}^{2}\right\rangle$ are larger than those of $2\left\langle\beta_{T} \widetilde{r}_{\text {out }} \widetilde{t}\right\rangle$. But at large $k_{T}$ the values of $2\left\langle\beta_{T} \widetilde{r}_{\text {out }} \widetilde{t}\right\rangle$ are close and are even larger than the corresponding results of $\left\langle\beta_{T}^{2} \widetilde{t}^{2}\right\rangle$. Both $\left\langle\beta_{T}^{2} \widetilde{t}^{2}\right\rangle$ and $2\left\langle\beta_{T} \widetilde{r}_{\text {out }} \widetilde{t}\right\rangle$ decrease with collision centrality. The near values of $\left\langle\beta_{T}^{2} \vec{t}^{2}\right\rangle$ and $2\left\langle\beta_{T} \widetilde{r}_{\text {out }} \tilde{t}\right\rangle$ at a fixed $k_{T}$ lead to the HBT results $R_{\text {out }} / R_{\text {side }} \approx 1$ for the granular sources. From Figures $8(\mathrm{e})$ and $8(\mathrm{f})$ one can see that the average transverse velocities of the pair at the RHIC energy are higher than those at the LHC energy at large $k_{T}$. However, the results of the average longitudinal velocities 


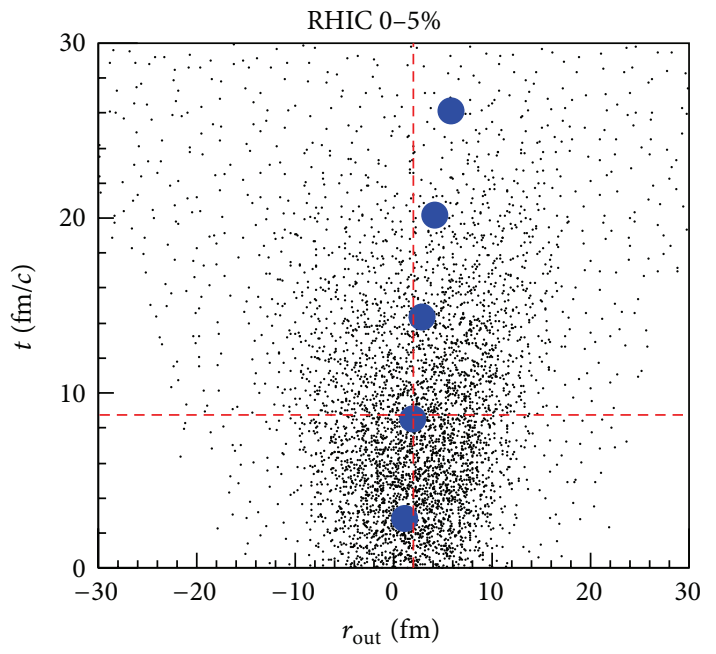

(a)

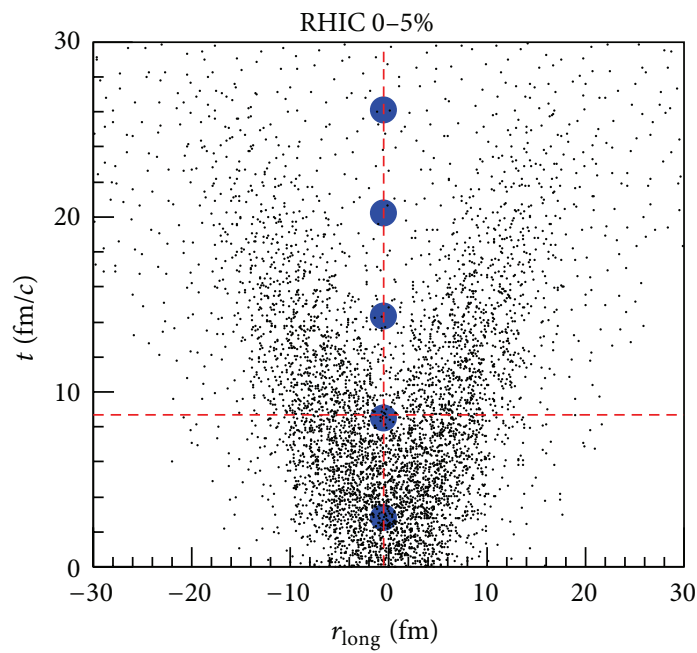

(c)

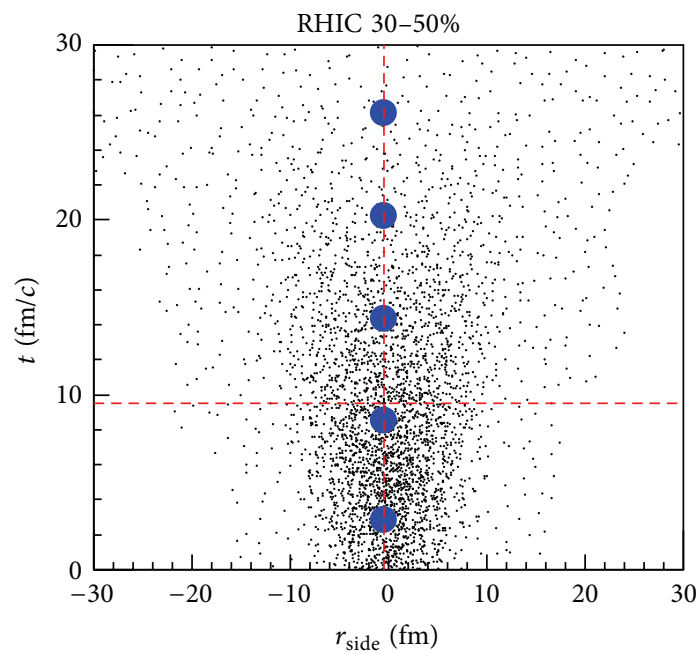

(e)

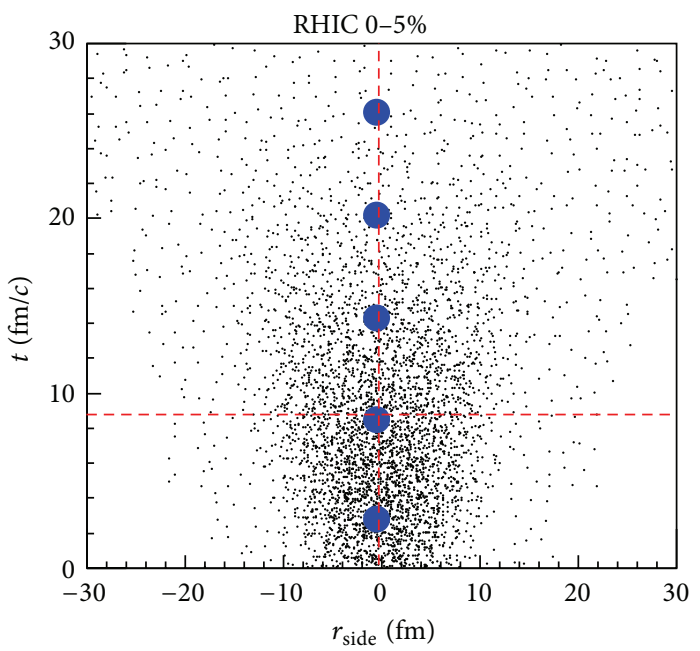

(b)

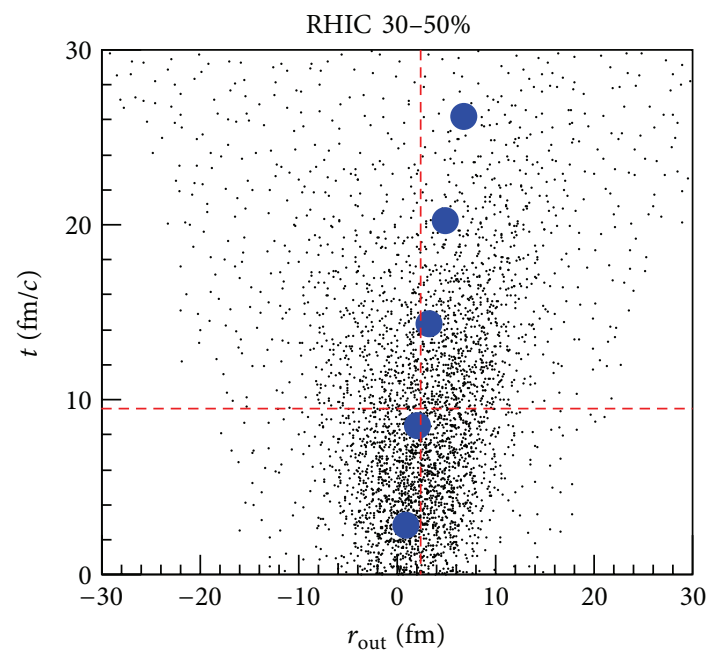

(d)

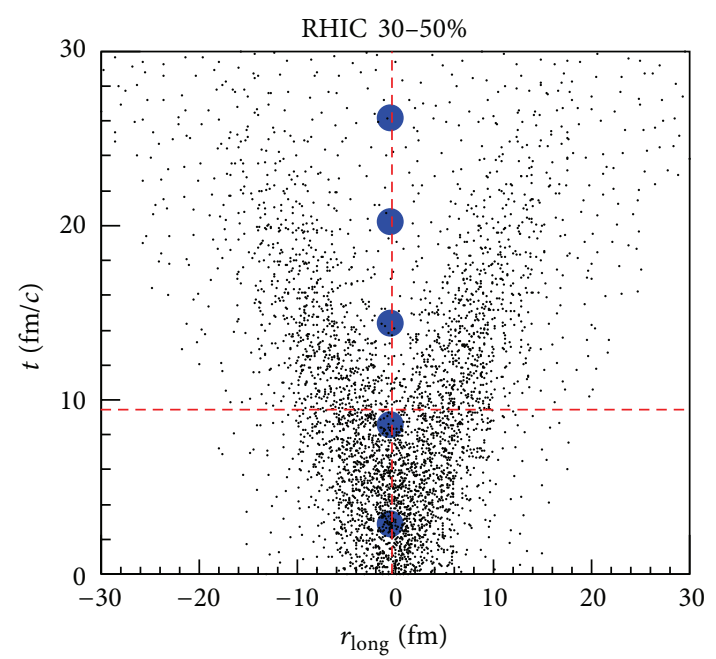

(f)

FIgURE 5: (Color online) the source-point distributions of final identical pions in $r_{i}-t$ plane ( $i=$ out, side, long), for the granular sources of the central and peripheral collisions at the RHIC energy. The dashed lines are the average values of $r_{i}$ and $t$ for all of the source points. The bullets are the average values of $r_{i}$ obtained from the same $t$ bins. 


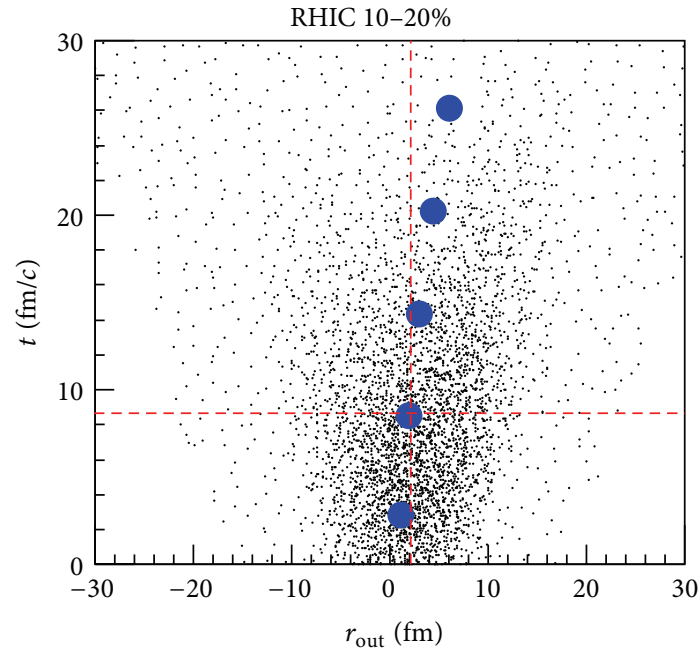

(a)

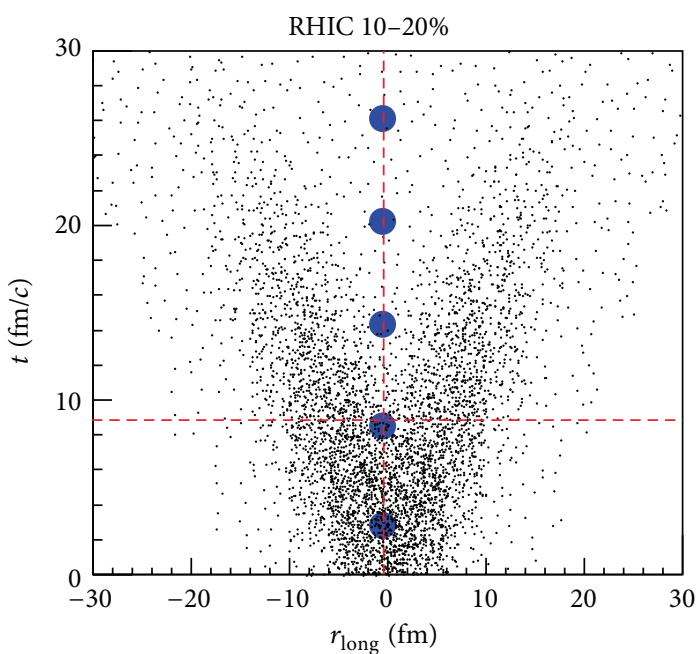

(c)

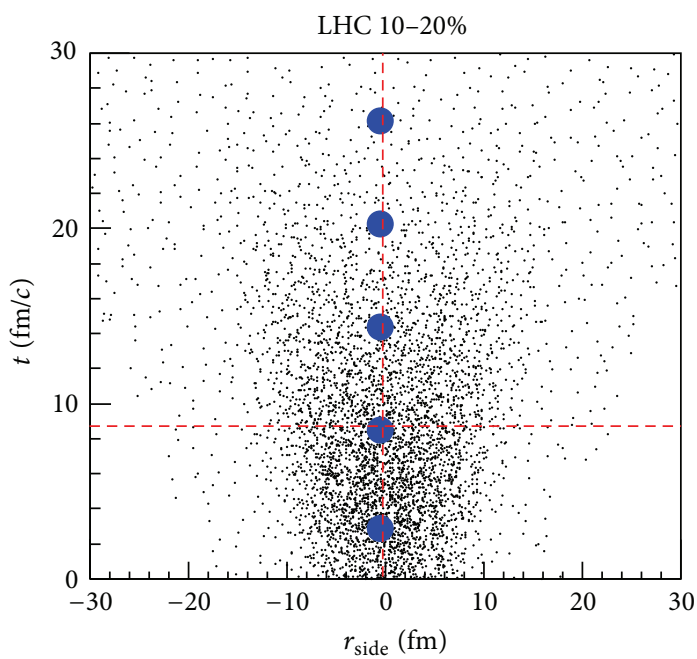

(e)

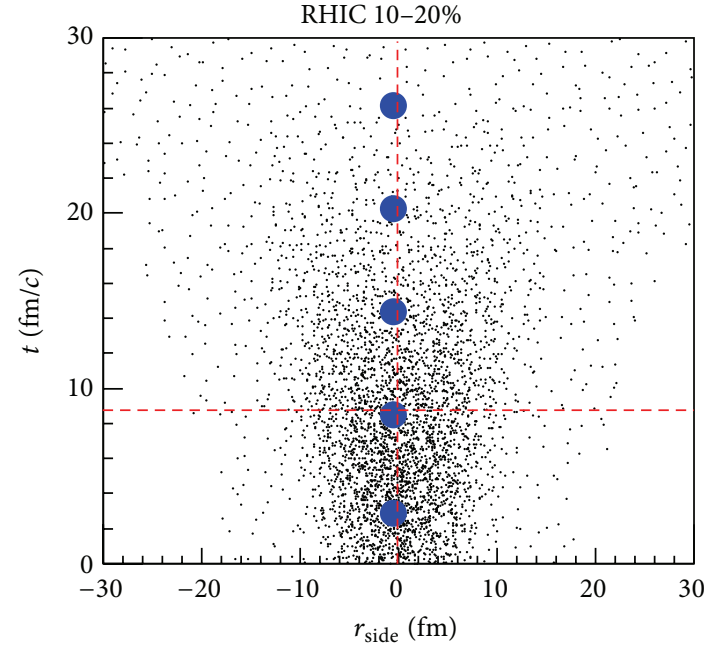

(b)

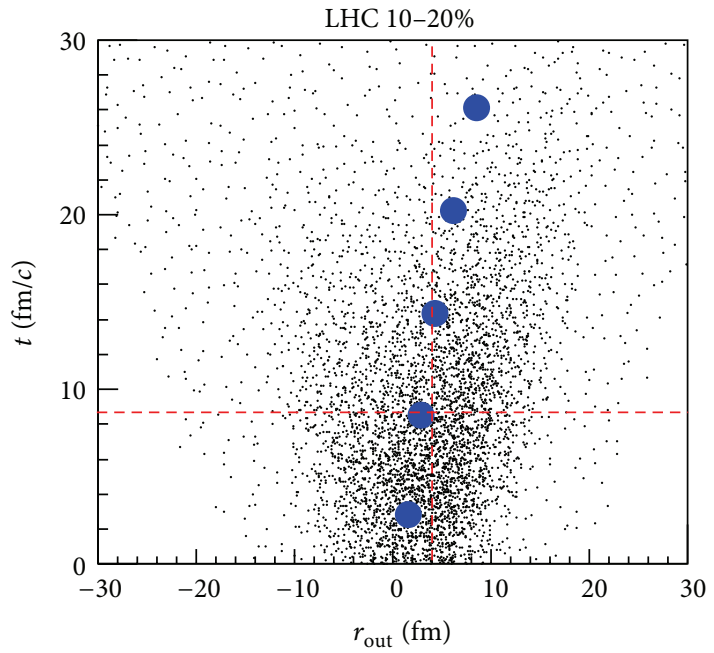

(d)

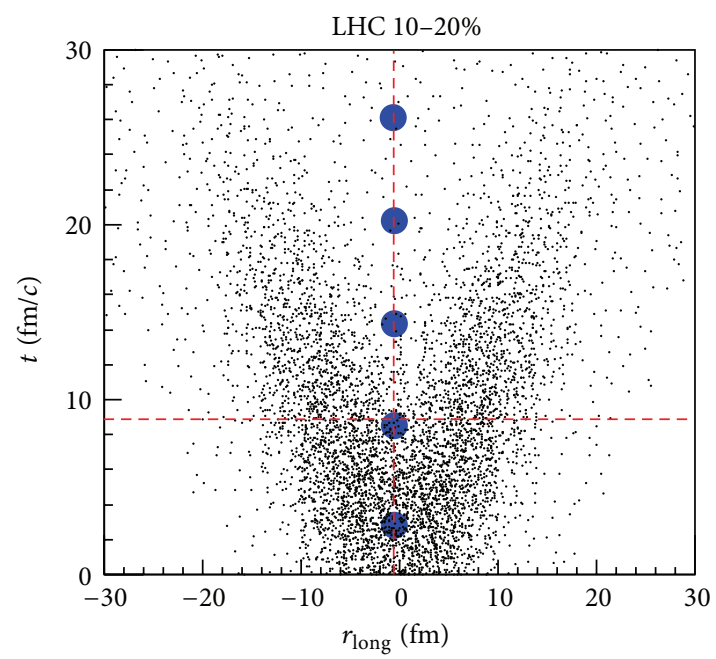

(f)

Figure 6: (Color online) the source-point distributions of final identical pions in $r_{i}-t$ plane ( $i=$ out, side, long), for the granular sources of the RHIC and LHC collisions with 10-20\% centrality. The dashed lines are the average values of $r_{i}$ and $t$ for all of the source points. The bullets are the average values of $r_{i}$ obtained from the same $t$ bins. 

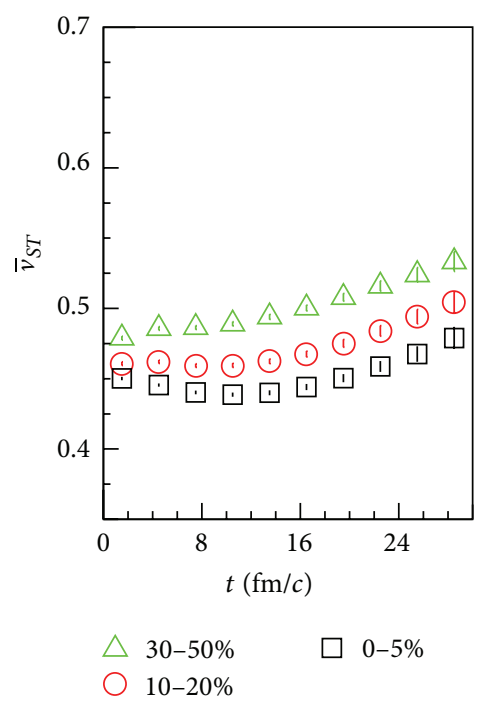

(a) RHIC

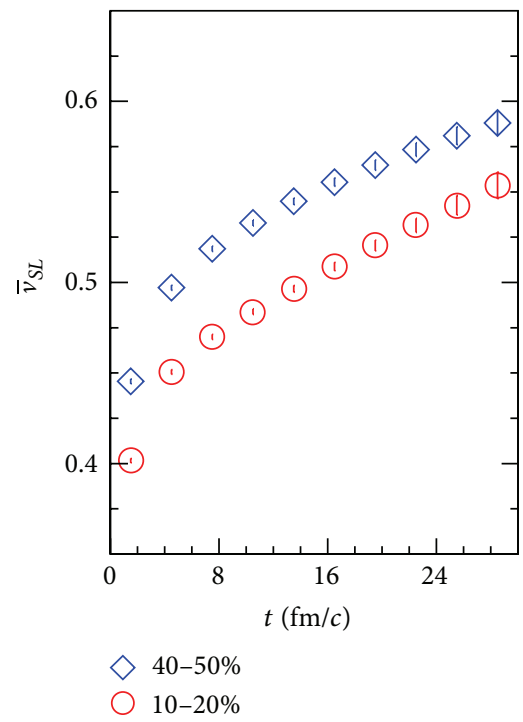

(d) LHC

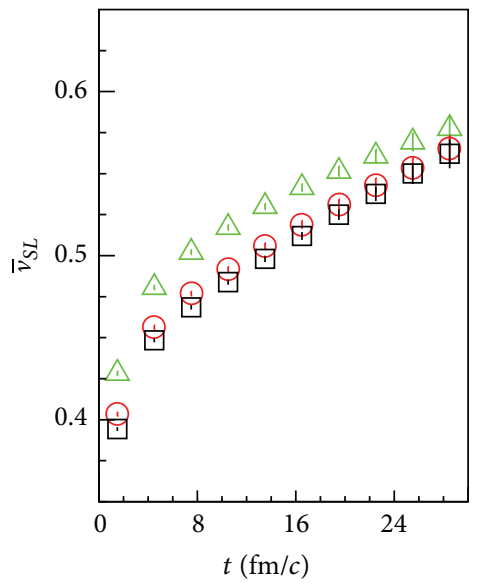

$\triangle 30-50 \% \quad \square 0-5 \%$

○ $10-20 \%$

(b) RHIC

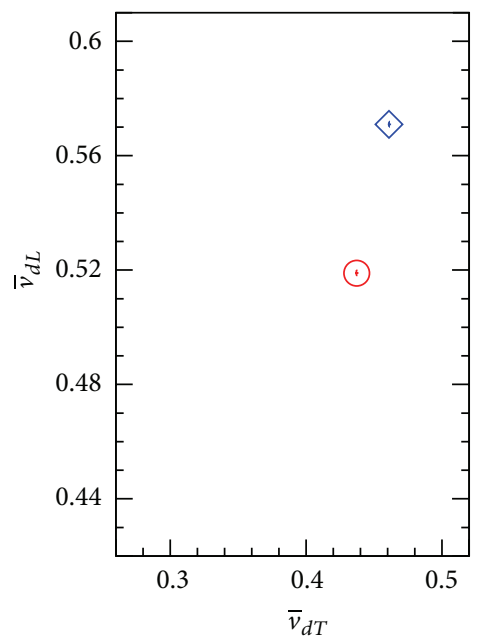

$\diamond 40-50 \%$

○ $10-20 \%$

(e) LHC

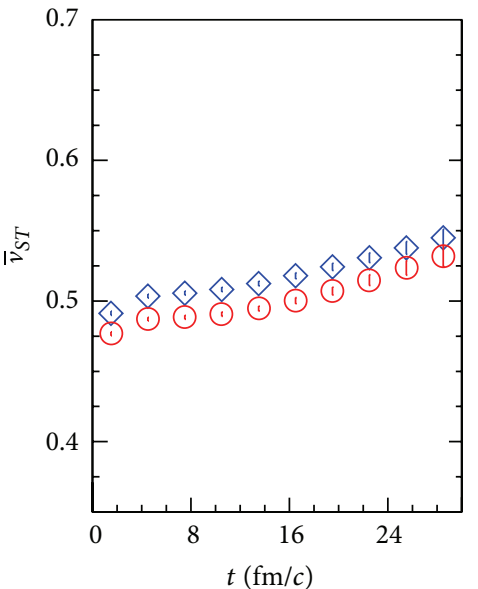

$\diamond 40-50 \%$

○ $10-20 \%$

(c) LHC

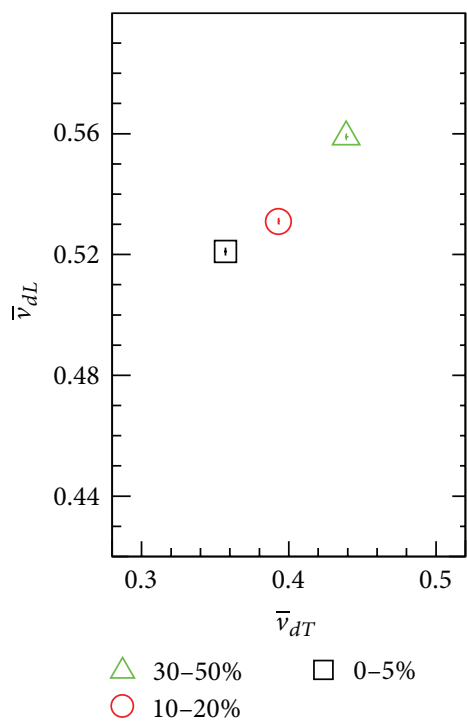

(f) RHIC

FIGURE 7: (Color online) ((a)-(d)) the average transverse and longitudinal velocities of the pion source points, $\bar{v}_{S T}$ and $\bar{v}_{S L}$, versus the emission time of the granular sources. ((e) and (f)) The average transverse and longitudinal droplet velocities $\bar{v}_{d T}$ and $\bar{v}_{d L}$ of the granular sources for the RHIC and LHC collisions.

are opposite. For a fixed $k_{T}$, a larger $\beta_{L}$ means a larger $k_{L}$ and therefore a larger $E_{k}\left(E_{k}^{2}=k_{T}^{2}+k_{L}^{2}+m_{k}^{2}\right)$ and smaller $\beta_{T}$. It will be seen that the reason for the larger $\bar{\beta}_{L}$ at large $k_{T}$ at the LHC energy is the larger longitudinal expansion of the granular source at the LHC energy as compared to that at the RHIC energy (see Figures 9(c) and 9(d)).

In Figure 9, we show the average emission time $\bar{t}=$ $\langle t\rangle\left(k_{T}\right)$, the time variance root $\Delta t=\left[\left\langle\tilde{t}^{2}\right\rangle\right]^{1 / 2}\left(k_{T}\right)$, and the average source transverse and longitudinal velocities $\bar{v}_{S T}\left(k_{T}\right)$ and $\bar{v}_{S L}\left(k_{T}\right)$ for the granular sources at the RHIC and LHC energies. The error bars for the results are statistical errors. One can see that the average emission time decreases with decreasing collision centrality and increases with increasing collision energy. It is because the average droplet velocity increases with decreasing collision centrality and increasing collision energy. The larger the droplet velocity, the larger time delay is. The values of $\Delta t$, which is also referred as the source lifetime, are almost independent of $k_{T}$ and smaller as compared to the values of $\bar{t}$. For a fixed $k_{T}$, the average source velocities increase with decreasing collision centrality. At large $k_{T}$, the average longitudinal velocities of the granular sources at the LHC energy are larger than the corresponding values at the RHIC energy. It is because the pion pairs with larger $k_{T}$ at the LHC energy correspond to a larger average emission time in the source center-of-mass frame (see Figure 9(b)) and therefore have larger average longitudinal source velocities as compared to those at the RHIC energy (see Figures $7(\mathrm{~b})$ and $7(\mathrm{~d})$ ). This result is consistent with the results of $\bar{\beta}_{L}$ shown in Figures $8(\mathrm{e})$ and $8(\mathrm{f})$. The large 


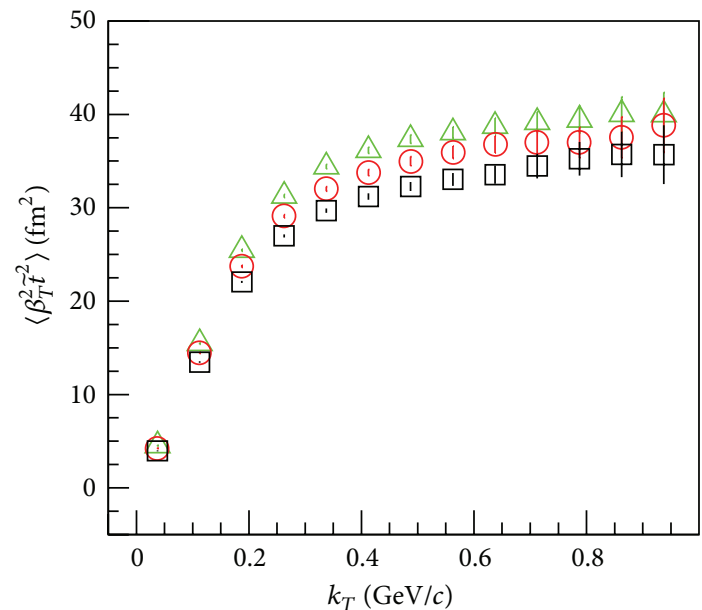

(a) RHIC

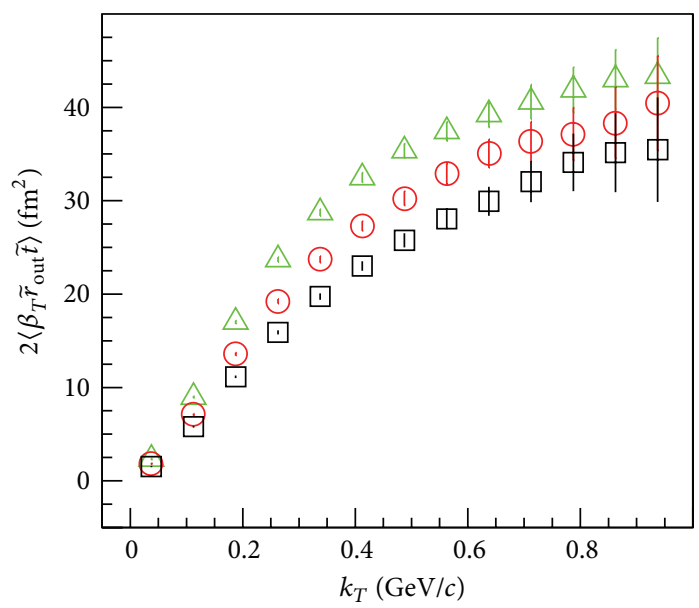

(c) RHIC

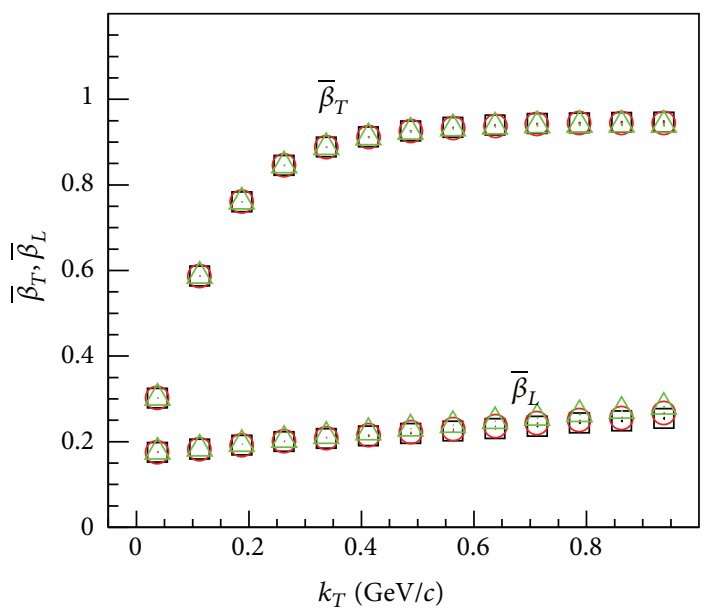

$\triangle 30-50 \%$

$\square 0-5 \%$

(e) RHIC

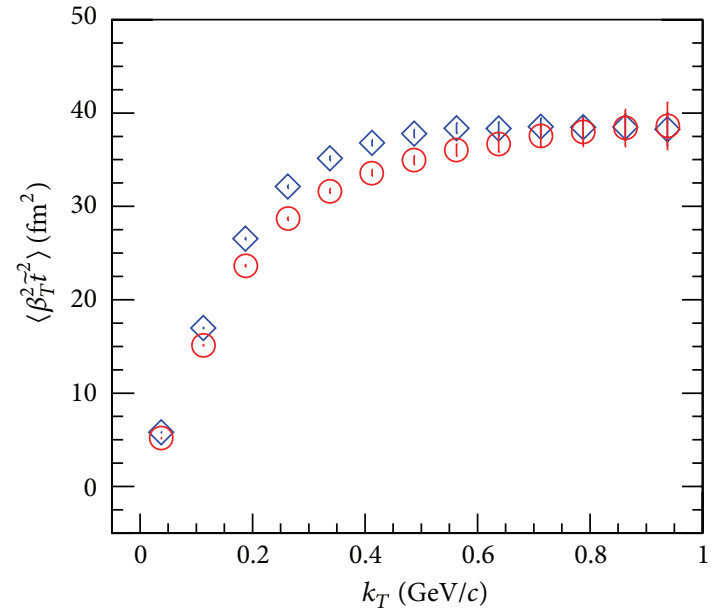

(b) LHC

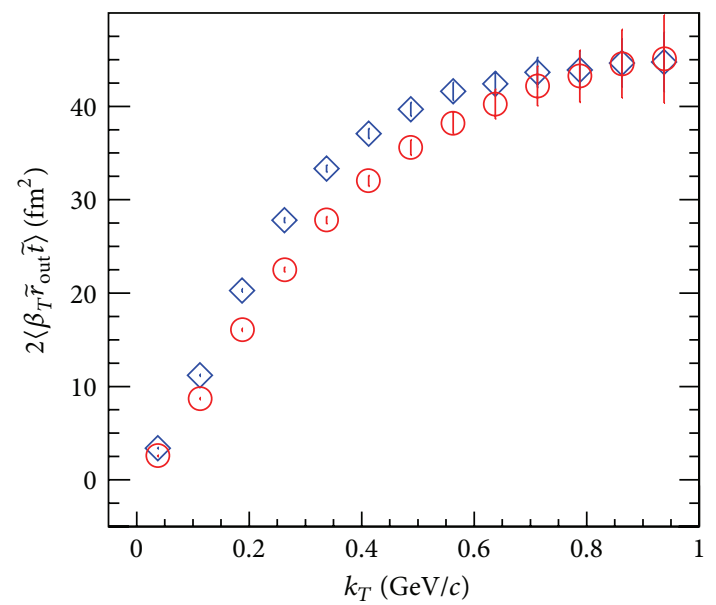

(d) LHC

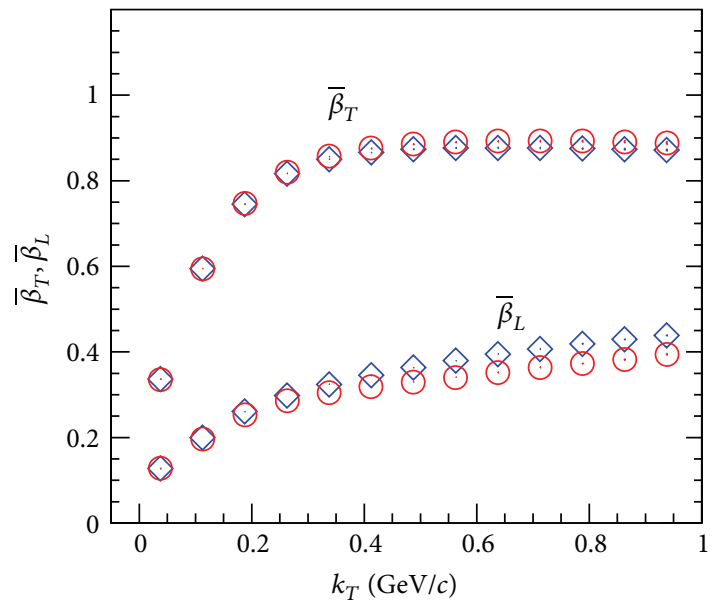

$\diamond 40-50 \%$

$10-20 \%$

(f) LHC

Figure 8: (Color online) the granular sources $\left\langle\beta_{T}^{2} \widetilde{t}^{2}\right\rangle, 2\left\langle\beta_{T} \widetilde{r}_{\text {out }} \widetilde{t}\right\rangle, \bar{\beta}_{T}$, and $\bar{\beta}_{L}$ versus $k_{T}$ for the RHIC and LHC collisions. 


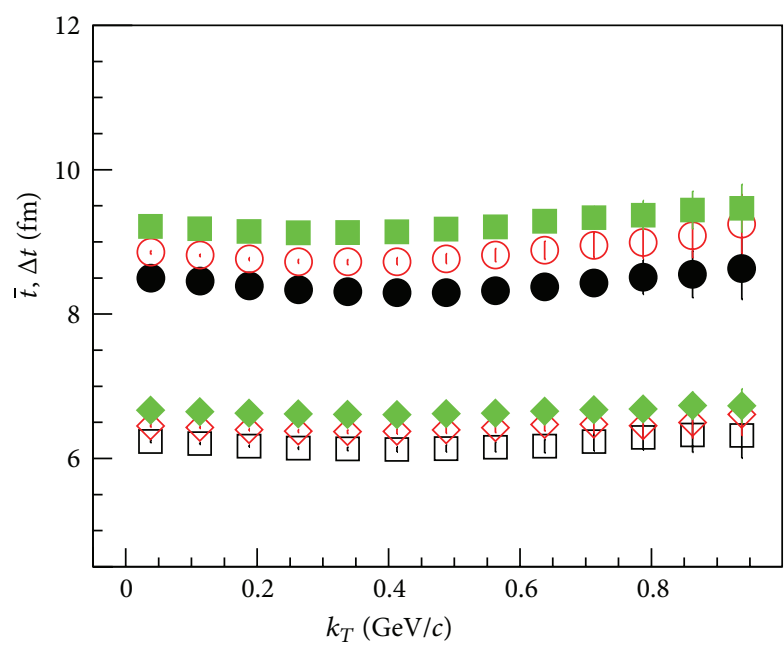

(a) RHIC

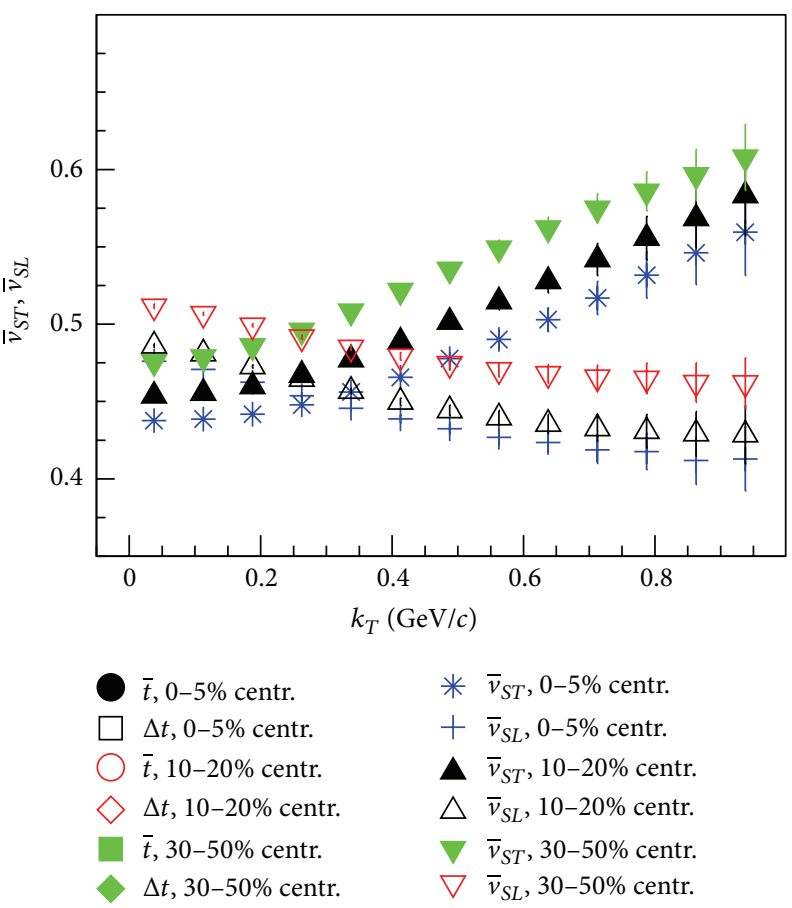

(c) RHIC

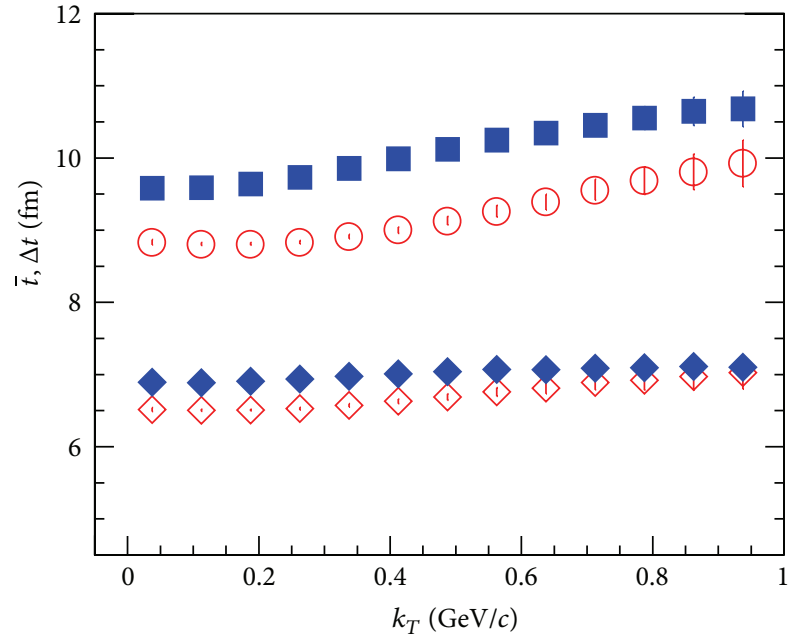

(b) LHC

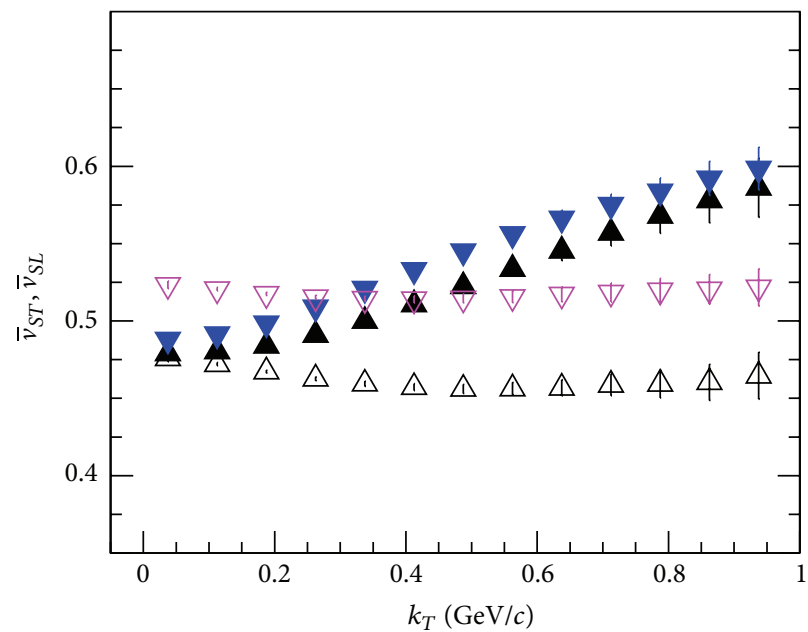

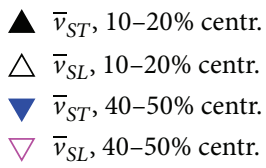

(d) LHC

FIGURE 9: (Color online) the $k_{T}$ dependence of the average emission time $\bar{t}=\langle t\rangle$, time variance root $\Delta t=\left[\left\langle\vec{t}^{2}\right\rangle\right]^{1 / 2}$, and average source transverse and longitudinal velocities $\bar{v}_{S T}$ and $\bar{v}_{S L}$ for the granular sources at the RHIC and LHC energies.

longitudinal velocity of the granular source at the LHC energy boosts strongly the pair momentum of the particles with almost the same emission direction and leads to the large $\bar{\beta}_{L}$ results.

Finally, it should be noted that the emission time mentioned in the paper is the time recorded from the initial state of the granular source. The real emission time from the beginning of collision should also include the system preequilibrium time $\tau_{0}$ and the breakup time $t_{0}$, which should be different for different collision energies [81] and centralities. However, the lifetime of the granular source is independent of the time original point. The small source lifetime is a character of the granular source $[64,65,81,100,101]$.

\section{Summary and Discussion}

We systemically investigate the pion transverse momentum spectrum, elliptic flow, and HBT interferometry in the granular source model for the heavy ion collisions at the RHIC highest energy and the LHC energy. The centrality and energy dependence of the observables at the two energies are examined. By comparing the granular source results with 
the experimental data of the Au-Au collisions at $\sqrt{s_{N N}}=$ $200 \mathrm{GeV}$ at the RHIC and the $\mathrm{Pb}-\mathrm{Pb}$ collisions at $\sqrt{s_{N N}}=$ $2.76 \mathrm{TeV}$ at the LHC with different collision centralities, we investigate the geometry and velocity parameters in the granular source model as a function of collision centrality and energy. The space-time structure and expansion velocities of the granular sources at the RHIC and LHC energies with different centralities are examined. Our investigations indicate that the granular source model can well reproduce the experimental data of pion transverse momentum spectra, elliptic flow, and HBT radii in the Au-Au collisions at $\sqrt{s_{N N}}=$ $200 \mathrm{GeV}$ with $0-5 \%, 10-20 \%$, and $30-50 \%$ centralities $[1,2,7$, $12]$ and in the $\mathrm{Pb}-\mathrm{Pb}$ collisions at $\sqrt{s_{N N}}=2.76 \mathrm{TeV}$ with $10-$ $20 \%$ and $40-50 \%$ centralities $[17,22,25]$. The experimental data of pion momentum spectra, elliptic flow, and HBT radii impose very strict constraints on the parameters in the granular source model. They exhibit certain regularities for collision centrality and energy. The space-time structure and expansion velocities of the granular source are consistent with that reflected by the observables.

In the granular source model we assume that the system created in the ultrarelativistic heavy ion collisions breaks up and forms the granular source of QGP droplets due to the dynamical instability in the fast expansion at the early stage and the surface tension of the strongly coupled QGP. We use ideal hydrodynamics to describe the droplet evolution and assume a Gaussian distribution for the droplet radius for simplicity. Because many droplets evolve simultaneously, the source lifetime is smaller as compared to that of a continued big source, which evolves in whole and freezes out from the source surface. For the granular source, the distribution of particle-emitting points of single event presents a clumpstructure during the source evolution. However, the distribution of source points for many events is continued and presents a volume distribution because of the contribution from the droplets in the central region of the source. The short source lifetime, clump-structure of source points distribution of single event, and volume distribution of source points for many events are the characters of the granular source.

The investigations for the granular source parameters indicate that the QGP droplets initially distribute in larger transverse and longitudinal regions for the more central and higher energy collisions. So, the distribution width of source points increases with increasing collision centrality and energy. In transverse direction, the droplet velocity of central collision is smaller than that of peripheral collision in the center region of the source, although the droplet transverse velocity of central collision is larger at the edge of the source. The average longitudinal velocity of droplet is larger than the average transverse velocity of droplet. The droplet longitudinal velocity increases with decreasing collision centrality. The larger droplet velocities in the peripheral collisions lead to larger average velocities of source. Because the difference of the droplet transverse velocities in and out of reaction plane decreases with collision centrality, the elliptic flow decreases with collision centrality. In HBT interferometry, the difference between the transverse HBT radii $R_{\text {out }}$ and $R_{\text {side }}$ is related to the transverse velocity of particle pair, the source lifetime, and the space-time correlation of source points. Both the quantities $\left\langle\beta_{T} \widetilde{t}^{2}\right\rangle$ and $2\left\langle\beta_{T} \widetilde{r}_{\text {out }} \widetilde{t}\right\rangle$ increase with the decreasing collision centrality and increasing transverse momentum of the pair, $k_{T}$. However, the difference between the two quantities for a fixed $k_{T}$ is approximately equal to zero. This leads to the results of $R_{\text {out }}\left(k_{T}\right) \approx R_{\text {side }}\left(k_{T}\right)$.

While one may argue on the details and the number of parameters used in the granular source model, the consistent explanation of a large number of measured one-particle and two-particle correlation quantities suggests that this model description captures some fundamental features of the spacetime dynamics. After all, it is a challenge to a model to explain the experimental data of the momentum spectra, elliptic flow, and HBT radii simultaneously at the RHIC and LHC energies, and the final criterion for a model is experiments. It will be of interest to improve the granular source model and investigate the effects of QGP viscosity and droplet interaction on the granular source parameters. On the other hand, the studies of the forming mechanism and signals of the granular source will be of interest.

\section{Conflict of Interests}

The authors declare that there is no conflict of interests regarding the publication of this paper.

\section{Acknowledgments}

The authors thank Dr. L. Cheng, Dr. U. Heinz, Dr. S. Jeon, and Dr. H. C. Song for helpful discussions. This research was supported by the National Natural Science Foundation of China under Grant no. 11275037.

\section{References}

[1] J. Adams, C. Adler, M. M. Aggarwal et al., "Identified particle distributions in pp and $\mathrm{Au}+\mathrm{Au}$ Collisions at $\sqrt{s_{N N}}=200 \mathrm{GeV}$," Physical Review Letters, vol. 92, no. 11, Article ID 112301, 6 pages, 2004.

[2] S. S. Adler, S. Afanasiev, C. Aidala et al., "Identified charged particle spectra and yields in $\mathrm{Au}+\mathrm{Au}$ collisions at $\sqrt{s_{N N}}=$ 200 GeV," Physical Review C, vol. 69, no. 3, Article ID 034909, 32 pages, 2004.

[3] B. B. Back, M. D. Baker, M. Ballintijn et al., "Identified hadron transverse momentum spectra in $\mathrm{Au}+\mathrm{Au}$ collisions at $\sqrt{s_{N N}}=$ 62.4 GeV,' Physical Review C, vol. 75, no. 2, Article ID 024910, 14 pages, 2007.

[4] C. Adler, Z. Ahammed, and C. Allgower, "Elliptic flow from two- and four-particle correlations in $\mathrm{Au}+\mathrm{Au}$ collisions at $\sqrt{s_{N N}}=130 \mathrm{GeV}$,' Physical Review C, vol. 66, no. 3, Article ID 034904, 17 pages, 2002.

[5] K. Adcox, S. S. Adler, and N. N. Ajitanand, "Flow measurements via two-particle azimuthal correlations in $\mathrm{Au}+\mathrm{Au}$ collisions at $\sqrt{s_{N N}}=130 \mathrm{GeV}$," Physical Review Letters, vol. 89, no. 21, Article ID 212301, 6 pages, 2002.

[6] S. S. Adler, S. Afanasiev, C. Aidala et al., "Elliptic flow of identified hadrons in Au+Au collisions at $\sqrt{s_{N N}}=200 \mathrm{GeV}$," Physical Review Letters, vol. 91, no. 18, Article ID 182301, 6 pages, 2003. 
[7] J. Adams, M. M. Aggarwal, and Z. Ahammed, "Azimuthal anisotropy in $\mathrm{Au}+\mathrm{Au}$ collisions at $\sqrt{\boldsymbol{s}_{N N}}=200 \mathrm{GeV}$," Physical Review Letters, vol. 72, no. 1, Article ID 014904, 23 pages, 2005.

[8] S. Afanasiev, C. Aidala, N. N. Ajitanand et al., "Systematic studies of elliptic flow measurements in $\mathrm{Au}+\mathrm{Au}$ collisions at $\sqrt{s_{N N}}=200 \mathrm{GeV}$," Physical Review C, vol. 80 , no. 2, Article ID 024909, 25 pages, 2009.

[9] C. Adler, Z. Ahammed, C. Allgower et al., "Pion interferometry of $\sqrt{s_{N N}}=200 \mathrm{GeV}$ Au+Au collisions at RHIC," Physical Review Letters, vol. 87, no. 8, Article ID 082301, 6 pages, 2001.

[10] K. Adcox, S. S. Adler, N. N. Ajitanand et al., "Transverse-mass dependence of two-pion correlations in $\mathrm{Au}+\mathrm{Au}$ collisions at $\sqrt{s_{N N}}=130$ GeV," Physical Review Letters, vol. 88, no. 19, Article ID 192302, 6 pages, 2002.

[11] S. S. Adler, S. Afanasiev, C. Aidala et al., "Bose-Einstein correlations of charged pion pairs in $\mathrm{Au}+\mathrm{Au}$ collisions at $\sqrt{s_{N N}}=$ 200 GeV," Physical Review Letters, vol. 93, no. 15, Article ID 152302, 6 pages, 2004.

[12] J. Adams, M. M. Aggarwal, Z. Ahammed et al., "Pion interferometry in Au+Au collisions at $\sqrt{s_{N N}}=200 \mathrm{GeV}$," Physical Review C, vol. 71, no. 4, Article ID 044906, 21 pages, 2005.

[13] S. S. Adler, S. Afanasiev, C. Aidala et al., "Evidence for a longrange component in the pion emission source in $\mathrm{Au}+\mathrm{Au}$ collisions at $\sqrt{s_{N N}}=200 \mathrm{GeV}$," Physical Review Letters, vol. 98, no. 13, Article ID 132301, 6 pages, 2007.

[14] S. Afanasiev, C. Aidala, N. N. Ajitanand et al., "Source breakup dynamics in $\mathrm{Au}+\mathrm{Au}$ collisions at $\sqrt{s_{N N}}=200 \mathrm{GeV}$ via threedimensional two-pion source imaging," Physical Review Letters, vol. 100, no. 23, Article ID 232301, 6 pages, 2008.

[15] M. Floris and ALICE Collaboration, "Identified particles in pp and $\mathrm{Pb}-\mathrm{Pb}$ collisions at LHC energies with the ALICE detector," Journal of Physics G: Nuclear and Particle Physics, vol. 38, no. 12, Article ID 124025, 2011.

[16] R. Preghenella and ALICE Collaboration, "Identified-particle production and spectra with the ALICE detector in $p p$ and $\mathrm{Pb}-\mathrm{Pb}$ collisions at the LHC," Acta Physica Polonica B, vol. 43, article 555, 2012.

[17] B. Abelev, J. Adam, D. Adamova et al., "Centrality dependence of $\pi, K$, and $p$ production in $\mathrm{Pb}-\mathrm{Pb}$ collisions at $\sqrt{s_{N N}}=$ 2.76 TeV," Physical Review C, vol. 88, no. 4, Article ID 044910, 23 pages, 2013.

[18] B. Abelev, J. Adam, D. Adamova et al., "Pion, Kaon, and proton production in central $\mathrm{Pb}-\mathrm{Pb}$ collisions at $\sqrt{s_{N N}}=2.76 \mathrm{TeV}$," Physical Review Letters, vol. 109, no. 25, Article ID 252301, 11 pages, 2012.

[19] B. Abelev, J. Adam, D. Adamova et al., "Centrality dependence of charged particle production at large transverse momentum in $\mathrm{Pb}-\mathrm{Pb}$ collisions at $\sqrt{s_{N N}}=2.76 \mathrm{TeV}$," Physics Letters B, vol. 720, no. 1-3, pp. 52-62, 2013.

[20] K. Aamodt, B. Abelev, A. A. Quintana et al., "Elliptic flow of charged particles in $\mathrm{Pb}-\mathrm{Pb}$ collisions at $\sqrt{s_{N N}}=2.76 \mathrm{TeV}$," Physical Review Letters, vol. 105, no. 25, Article ID 252302, 11 pages, 2010.

[21] K. Aamodt, B. Abelev, A. A. Quintana et al., "Higher harmonic anisotropic flow measurements of charged particles in $\mathrm{Pb}-\mathrm{Pb}$ collisions at $\sqrt{s_{N N}}=2.76 \mathrm{TeV}$," Physical Review Letters, vol. 107, no. 3, Article ID 032301, 10 pages, 2011.

[22] R. Snellings and ALICE Collaboration, "Anisotropic flow at the LHC measured with the ALICE detector," Journal of Physics G, vol. 38, no. 12, Article ID 124013, 2011.
[23] M. Krzewicki and ALICE Collaboration, "Elliptic and triangular flow of identified particles measured with the ALICE detector at the LHC," Journal of Physics G: Nuclear and Particle Physics, vol. 38, no. 12, Article ID 124047, 2011.

[24] K. Aamodt, A. Abrahantes Quintanab, D. Adamová et al., “Twopion Bose-Einstein correlations in central $\mathrm{Pb}-\mathrm{Pb}$ collisions $\sqrt{s_{N N}}=2.76$ TeV," Physics Letters B, vol. 696, no. 4, pp. 328-337, 2011.

[25] A. Kisiel and ALICE Collaboration, "Overview of the femtoscopy of $\mathrm{Pb}-\mathrm{Pb}$ and pp collisions at the LHC with the ALICE experiment," in Proceedings of the 7th Workshop on Particle Correlations and Femtoscopy (WPCF '11), PoS no. 003, Tokyo, Japan, September 2011.

[26] B.-C. Li, Y.-Y. Fu, L.-L. Wang, and F.-H. Liu, “Transverse momentum, centrality, and participant nucleon number dependence of elliptic flow," Advances in High Energy Physics, vol. 2013, Article ID 908046, 7 pages, 2013.

[27] Y. M. Sinyukov, S. V. Akkelin, I. A. Karpenko, and V. M. Shapoval, "Femtoscopic and nonfemtoscopic two-particle correlations in $A+A$ and $P+P$ collisions at RHIC and LHC energies," Advances in High Energy Physics, vol. 2013, Article ID 198928, 23 pages, 2013.

[28] F.-H. Liu, Y.-H. Chen, H.-R. Wei, and B.-C. Li, "Transverse momentum distributions of final-state particles produced in soft excitation process in high energy collisions," Advances in High Energy Physics, vol. 2013, Article ID 965735, 15 pages, 2013.

[29] I. Arsene, I. G. Bearden, D. Beavis et al., "Quark-gluon plasma and color glass condensate at RHIC? The perspective from the BRAHMS experiment," Nuclear Physics A, vol. 757, no. 1-2, pp. $1-27,2005$.

[30] B. B. Backa, M. D. Bakerb, M. Ballintijnd et al., “The PHOBOS perspective on discoveries at RHIC," Nuclear Physics A, vol. 757, no. 1-2, pp. 28-101, 2005.

[31] J. Adams, M. M. Aggarwalac, Z. Ahammed et al., "Experimental and theoretical challenges in the search for the quark-gluon plasma: The STAR Collaboration's critical assessment of the evidence from RHIC collisions," Nuclear Physics A, vol. 757, no. 1-2, pp. 102-183, 2005.

[32] K. Adcoxbd, S. S. Adlere, S. Afanasiev et al., "Formation of dense partonic matter in relativistic nucleus-nucleus collisions at RHIC: experimental evaluation by the PHENIX Collaboration," Nuclear Physics A, vol. 757, no. 1-2, pp. 184-283, 2005.

[33] J. Lajoie, "PHENIX highlights I: propagation of partons in a coloured medium," Journal of Physics G: Nuclear and Particle Physics, vol. 34, no. 8, pp. S191-S198, 2007.

[34] L. Ruan, "Physics with identified particles at STAR," Journal of Physics G: Nuclear and Particle Physics, vol. 34, no. 8, pp. S199S206, 2007.

[35] I. G. Bearden and BRAHMS Collaboration, "Recent highlights from BRAHMS," Journal of Physics G: Nuclear and Particle Physics, vol. 34, no. 8, pp. S207-S216, 2007.

[36] D. J. Hofman, "Latest results from PHOBOS," Journal of Physics G: Nuclear and Particle Physics, vol. 34, no. 8, pp. S217-S224, 2007.

[37] M. C. de la Barca Sánchez, "STAR results on high transverse momentum, heavy flavour and electromagnetic probes," Journal of Physics G: Nuclear and Particle Physics, vol. 34, no. 8, pp. S225-S232, 2007.

[38] J.-P. Blaizot, "Theoretical overview: towards understanding the quark-gluon plasma," Journal of Physics G: Nuclear and Particle Physics, vol. 34, no. 8, pp. S243-S253, 2007. 
[39] L. McLerran, “Theory summary: quark matter 2006," Journal of Physics G: Nuclear and Particle Physics, vol. 34, no. 8, pp. S583S592, 2007.

[40] A. Franz and PHENIX Collaboration, "Highlights from PHENIX: I," Journal of Physics G: Nuclear and Particle Physics, vol. 35, no. 10, Article ID 104002, 2008.

[41] R. Debbe and BRAHMS Collaboration, "Recent results from BRAHMS," Journal of Physics G: Nuclear and Particle Physics, vol. 35, no. 10, Article ID 104004, 2008.

[42] B. Wosiek, M. Baker, D. Barton et al., "Latest results from the PHOBOS experiment," Journal of Physics G: Nuclear and Particle Physics, vol. 35, no. 10, Article ID 104005, 2008.

[43] B. Mohanty and STAR Collaboration, "STAR results on medium properties and response of the medium to energetic partons," Journal of Physics G: Nuclear and Particle Physics, vol. 35, no. 10, Article ID 104006, 2008.

[44] T. C. Awes and PHENIX Collaboration, "Highlights from PHENIX: II," Journal of Physics G, vol. 35, no. 10, Article ID 104007, 2008.

[45] L. McLerran, "From AGS-SPS and onwards to the LHC," Journal of Physics G: Nuclear and Particle Physics, vol. 35, no. 10, Article ID 104001, 2008.

[46] R. Venugopalan, "From glasma to quark-gluon plasma in heavy-ion collisions," Journal of Physics G: Nuclear and Particle Physics, vol. 35, Article ID 104003, 2008.

[47] R. K. Seto, "Summary of experimental results: photons, leptons and heavy quarks," Journal of Physics G: Nuclear and Particle Physics, vol. 35, no. 10, Article ID 104043, 2008.

[48] E. Shuryak, "Quark-gluon plasma-new frontiers," Journal of Physics G: Nuclear and Particle Physics, vol. 35, no. 10, Article ID 104044, 2008.

[49] W. A. Zajc, "Puzzles, progress, prospects: pre-summary for the Quark Matter 2009 Conference," Nuclear Physics A, vol. 830, no. 1-4, pp. 3c-10c, 2009.

[50] S. Bathe and PHENIX Collaboration, "PHENIX highlights," Journal of Physics G: Nuclear and Particle Physics, vol. 38, no. 12, Article ID 124001, 2011.

[51] H. Masui and STAR Collaboration, "STAR highlights," Journal of Physics G: Nuclear and Particle Physics, vol. 38, no. 12, Article ID 124002, 2011.

[52] S. Esumi and PHENIX Collaboration, "Collective flow measurements from the PHENIX experiment," Journal of Physics G: Nuclear and Particle Physics, vol. 38, no. 12, Article ID 124010, 2011.

[53] P. Sorensen and STAR Collaboration, "Higher flow harmonics in heavy ion collisions from STAR," Journal of Physics G: Nuclear and Particle Physics, vol. 38, no. 12, Article ID 124029, 2011.

[54] B. Schenke, "Flow in heavy-ion collisions-theory perspective," Journal of Physics G: Nuclear and Particle Physics, vol. 38, Article ID 124009, 2011.

[55] F. Antinori, "The high energy frontier of ultrarelativistic nucleus-nucleus collisions," Journal of Physics G: Nuclear and Particle Physics, vol. 38, no. 12, Article ID 124038, 2011.

[56] U. A. Wiedemann, "Introductory overview of quark matter 2012," Nuclear Physics A, vol. 904-905, pp. 3c-10c, 2013.

[57] B. Hippolyte and D. H. Rischke, "Global variables and correlations: summary of the results presented at the Quark Matter 2012 conference," Nuclear Physics A, vol. 904-905, pp. 318c-325c, 2013.

[58] K. Aamodt, A. A. Quintana, D. Adamova et al., "Centrality dependence of the charged-particle multiplicity density at midrapidity in $\mathrm{Pb}-\mathrm{Pb}$ collisions at $\sqrt{{s_{N N}}}=2.76 \mathrm{TeV}$," Physical Review Letters, vol. 106, Article ID 032301, 2011.

[59] J. Velkovska and CMS Collaboration, "Anisotropic flow measurements in $\sqrt{\mathbf{s}_{\mathrm{NN}}}=2.76 \mathrm{TeV} \mathrm{Pb}-\mathrm{Pb}$ collisions from the CMS experiment," Journal of Physics G, vol. 38, Article ID 124011, 2011.

[60] K. Aamodt, B. Abelevb, A. Abrahantes Quintana et al., "Harmonic decomposition of two particle angular correlations in $\mathrm{Pb}-\mathrm{Pb}$ collisions at $\sqrt{s_{N N}}=2.76$," Physics Letters B, vol. 708, pp. 249-264, 2012.

[61] K. Šafarík and ALICE Collaboration, "Overview of recent ALICE results," Nuclear Physics A, vol. 904-905, pp. 27c-34c, 2013.

[62] B. Wosiek and ATLAS Collaboration, "ATLAS results from leadlead collisions at $\sqrt{s_{N N}}=2.76 \mathrm{TeV}$," Nuclear Physics A, vol. 904905, pp. 35c-42c, 2013.

[63] S. J. Sanders and CMS Collaboration, "Overview of results on flow and correlations from the CMS Collaboration," Nuclear Physics A, vol. 904-905, pp. 98c-105c, 2013.

[64] W. N. Zhang, M. J. Efaaf, and C. Y. Wong, "Pion interferometry for a granular source of quark-gluon plasma droplets," Physical Review C, vol. 70, Article ID 024903, 2004.

[65] W. N. Zhang, Y. Y. Ren, and C. Y. Wong, "Analysis of pion elliptic flow and Hanbury-Brown-Twiss interferometry in a granular quark-gluon plasma droplet model," Physical Review C, vol. 74, Article ID 024908, 2006.

[66] W. N. Zhang, Z. T. Yang, and Y. Y. Ren, "Characteristic quantities of pion-emitting sources extracted by model-independent analysis in relativistic heavy ion collisions," Physical Review C, vol. 80, Article ID 044908, 2009.

[67] Z. T. Yang, W. N. Zhang, L. Huo, J. B. Zhang, and J. Phys, "Imaging of granular sources in high energy heavy ion collisions," Journal of Physics G, vol. 36, no. 1, Article ID 015113, 2009.

[68] G. Bertsch, M. Gong, and M. Tohyama, "Pion interferometry in ultrarelativistic heavy-ion collisions," Physical Review C, vol. 37, no. 5, pp. 1896-1900, 1988.

[69] G. F. Bertsch, "Pion interferometry as a probe of the plasma," Nuclear Physics A, vol. 498, pp. 173-179, 1989.

[70] S. Pratt, T. Csörgo, and J. Zimányi, "Detailed predictions for two-pion correlations in ultrarelativistic heavy-ion collisions," Physical Review C, vol. 42, p. 2646, 1990.

[71] D. A. Brown and P. Danielewicz, "Imaging of sources in heavyion reactions," Physics Letters B, vol. 398, pp. 252-258, 1997.

[72] D. A. Brown and P. Danielewicz, "Optimized discretization of sources imaged in heavy-ion reactions," Physical Review CNuclear Physics, vol. 57, no. 5, pp. 2474-2483, 1998.

[73] D. A. Brown and P. Danielewicz, "Observing non-Gaussian sources in heavy-ion reactions," Physical Review C, vol. 64, Article ID 014902, 2001.

[74] P. Danielewicz and S. Pratt, "Analysis of low-momentum correlations with Cartesian harmonics," Physics Letters, Section B: Nuclear, Elementary Particle and High-Energy Physics, vol. 618, no. 1-4, pp. 60-67, 2005.

[75] P. Danielewicz and S. Pratt, "Analyzing correlation functions with tesseral and Cartesian spherical harmonics," Physical Review C, vol. 75, Article ID 034907, 2007.

[76] C. Y. Wong and W. N. Zhang, "Signature of granular structures by single-event intensity interferometry," Physical Review C, vol. 70, Article ID 064904, 2004.

[77] W. N. Zhang, S. X. Li, C. Y. Wong, and M. J. Efaaf, "Signals in single-event pion interferometry for granular sources of quarkgluon plasma droplets," Physical Review C, vol. 71, Article ID 064908, 2005. 
[78] Y. Y. Ren, W. N. Zhang, and J. L. Liu, "Detection of source inhomogeneity through event-by-event two-pion Bose-Einstein correlations," Physics Letters B, vol. 669, pp. 317-320, 2008.

[79] C. E. Aguiar, T. Kodama, T. Osada, and Y. Hama, "Smoothed particle hydrodynamics for relativistic heavy-ion collisions," Journal of Physics G: Nuclear and Particle Physics, vol. 27, no. 1, pp. 75-94, 2001.

[80] Y. Hama, T. Kodama, and O. Socolowski Jr., "Topics on hydrodynamic model of nucleus-nucleus collisions," Brazilian Journal of Physics, vol. 35, no. 1, p. 24, 2005.

[81] W. N. Zhang, H. J. Yin, and Y. Y. Ren, "Two-pion interferometry for the granular sources in ultrarelativistic heavy ion collisions at the RHIC and the LHC", Chinese Physics Letters, vol. 28, no. 12, Article ID 122501, 2011.

[82] A. Adare, M. Luzum, and H. Petersen, "Initial state fluctuations and final state correlations: status and open questions," Physica Scripta, vol. 87, no. 4, Article ID 048001, 2013.

[83] J. Takahashi, B. M. Tavares, W. L. Qian et al., "Topology studies of hydrodynamics using two-particle correlation analysis," Physical Review Letters, vol. 103, Article ID 242301, 2009.

[84] C. Y. Wong, "Toroidal and spherical bubble nuclei," Annals of Physics, vol. 77, no. 1-2, pp. 279-353, 1973.

[85] G. Torrieri, B. Tomášik, and I. Mishustin, "Bulk-viscositydriven clusterization of quark-gluon plasma and early freezeout in relativistic heavy-ion collisions," Physical Review C, vol. 77, Article ID 034903, 2008.

[86] D. J. Bjorken, "Highly relativistic nucleus-nucleus collisions: the central rapidity region," Physical Review D, vol. 27, no. 1, pp. 140151, 1983.

[87] C. Shen, U. Heinz, P. Huovinen, and H. C. Song, "Systematic parameter study of hadron spectra and elliptic flow from viscous hydrodynamic simulations of $\mathrm{Au}+\mathrm{Au}$ collisions at $\sqrt{\bar{s}_{N N}}=$ 200 GeV," Physical Review C, vol. 82, Article ID 054904, 2010.

[88] S. Voloshin and Y. Zhang, "Flow study in relativistic nuclear collisions by Fourier expansion of azimuthal particle distributions," Zeitschrift für Physik C Particles and Fields, vol. 70, no. 4, pp. 665-671, 1996.

[89] A. M. Poskanzer and S. A. Voloshin, "Methods for analyzing anisotropic flow in relativistic nuclear collisions," Physical Review C-Nuclear Physics, vol. 58, no. 3, pp. 1671-1678, 1998.

[90] N. Borghini, P. M. Dinh, and J. Y. Ollitrault, "Flow analysis from multiparticle azimuthal correlations," Physical Review C, vol. 64, Article ID 054901, 2001.

[91] R. S. Bhalerao and J.-Y. Ollitrault, "Eccentricity fluctuations and elliptic flow at RHIC," Physics Letters, Section B: Nuclear, Elementary Particle and High-Energy Physics, vol. 641, no. 3-4, pp. 260-264, 2006.

[92] M. Gyulassy, S. K. Kauffmann, and L. W. Wilson, "Pion interferometry of nuclear collisions. I. Theory," Physical Review C, vol. 20, no. 6, pp. 2267-2292, 1979.

[93] C. Y. Wong, Introduction to High-Energy Heavy-Ion Collisions, chapter 17, World Scientific, Singapore, 1994.

[94] U. A. Wienemann and U. Heinz, "Particle interferometry for relativistic heavy-ion collisions," Physics Reports, vol. 319, no. 45, pp. 145-230, 1999.

[95] R. M. Weiner, "Boson interferometry in high-energy physics," Physics Reports, vol. 327, no. 5, pp. 249-346, 2000.

[96] M. A. Lisa, S. Pratt, R. Soltz, and U. Wiedemann, "Femtoscopy in relativistic heavy ion collisions: two decades of progress," Annual Review of Nuclear and Particle Science, vol. 55, pp. 357402, 2005.
[97] P. F. Kolb, P. Huovinen, U. Heinz, and H. Heiselberg, "Elliptic flow at SPS and RHIC: from kinetic transport to hydrodynamics," Physics Letters, Section B: Nuclear, Elementary Particle and High-Energy Physics, vol. 500, no. 3-4, pp. 232-240, 2001.

[98] T. Hirano and K. Tsuda, "Collective flow and two-pion correlations from a relativistic hydrodynamic model with early chemical freeze-out," Physical Review C, vol. 66, no. 5, Article ID 054905, 2002.

[99] L. G. Pang, Q. Wang, and X. N. Wang, "Effects of initial flow velocity fluctuation in event-by-event $(3+1) D$ hydrodynamics," Physical Review C, vol. 86, Article ID 024911, 2012.

[100] W.-N. Zhang and C.-Y. Wong, "Explanation of the RHIC HBT puzzle by a granular source of quark-gluon plasma droplets," International Journal of Modern Physics E, vol. 16, no. 10, pp. 3262-3270, 2007.

[101] W.-N. Zhang, "Two-pion interferometry for the granular sources in the heavy ion collisions at RHIC and LHC energies," in Proceedings of the 7th Workshop on Particle Correlations and Femtoscopy (WPCF '11), PoS no. 051, Tokyo, Japan, September 2011.

[102] M. Herrmann and G. F. Bertsch, "Source dimensions in ultrarelativistic heavy-ion collisions," Physical Review C, vol. 51, no. 1, pp. 328-338, 1995.

[103] S. Chapman, P. Scotto, and U. Heinz, "New cross term in the two-particle Hanbury-Brown-Twiss correlation function in ultrarelativistic heavy-ion collisions," Physical Review Letters, vol. 74, article 4400, 1995. 

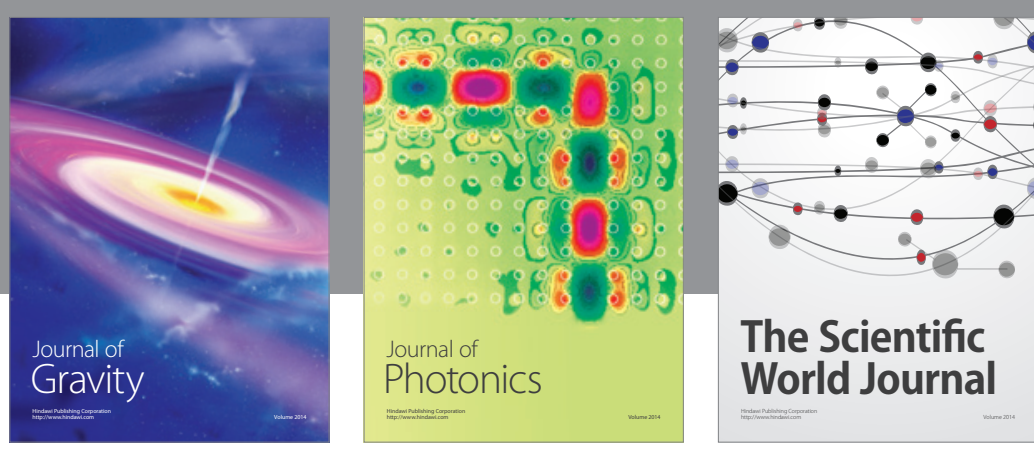

The Scientific World Journal
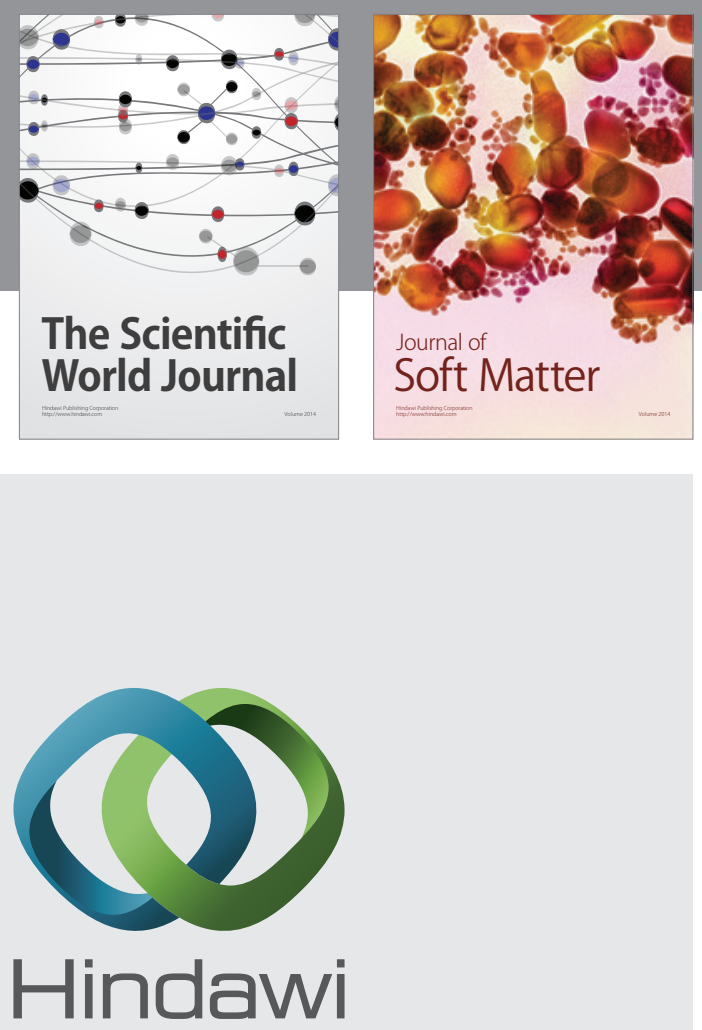

Submit your manuscripts at

http://www.hindawi.com

nternational Journal of

Statistical Mechanics
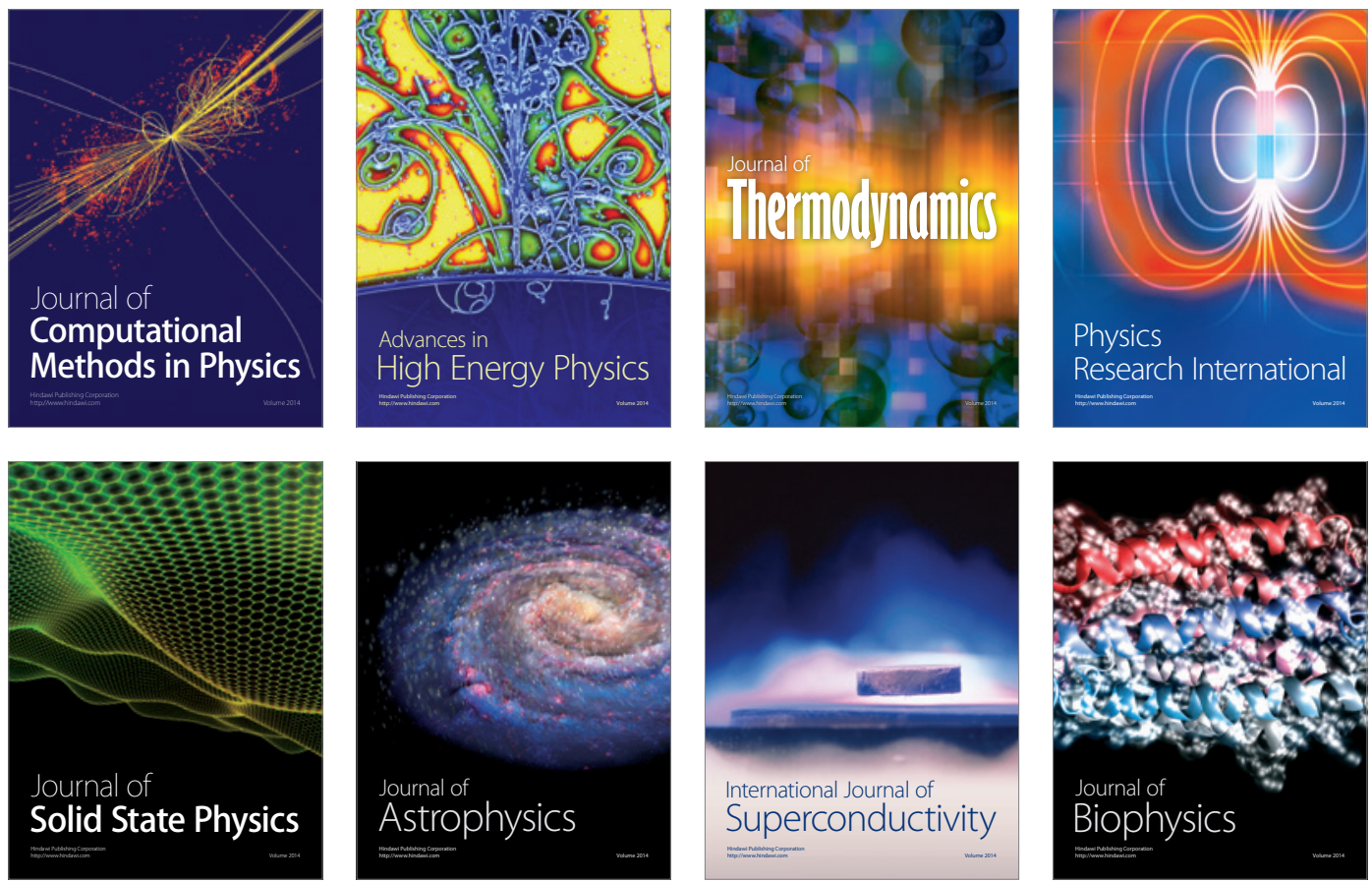
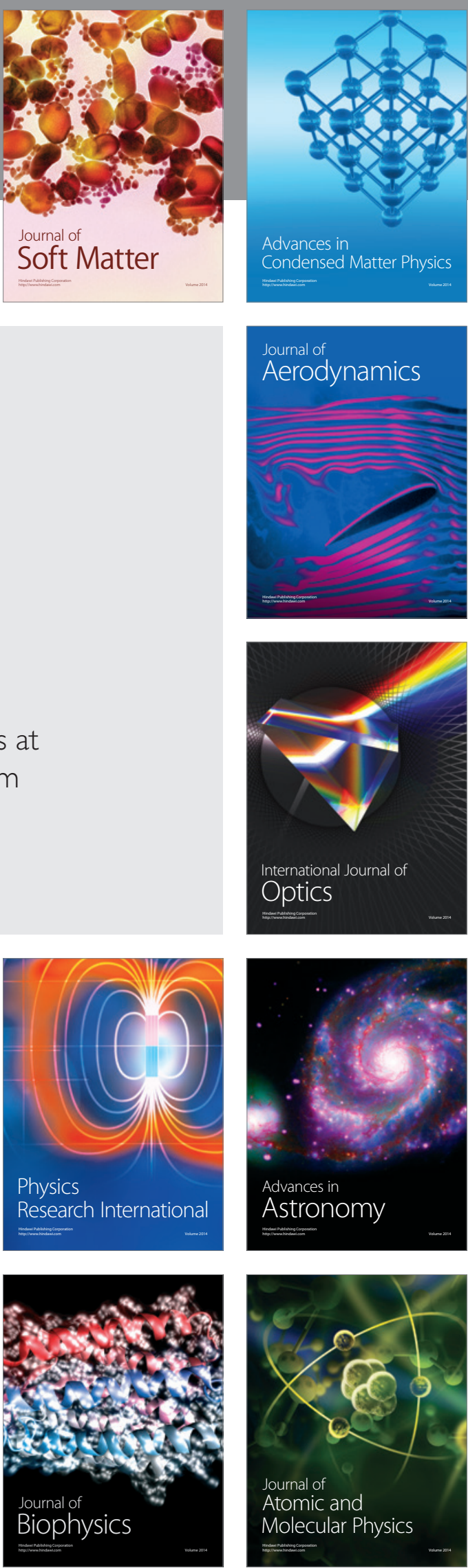